Purdue University Purdue e-Pubs

2012

\title{
The Low Frequency Performance of Metamaterial Barriers Based on Cellular Structures
}

J Stuart Bolton

Purdue University, bolton@purdue.edu

Srinivas Varanasi

Thomas Siegmund

Purdue University, siegmund@purdue.edu

Ray Cipra

Purdue University, cipra@purdue.edu

Follow this and additional works at: http:// docs.lib.purdue.edu/herrick

Bolton, J Stuart; Varanasi, Srinivas; Siegmund, Thomas; and Cipra, Ray, "The Low Frequency Performance of Metamaterial Barriers Based on Cellular Structures" (2012). Publications of the Ray W. Herrick Laboratories. Paper 51.

http://docs.lib.purdue.edu/herrick/51

This document has been made available through Purdue e-Pubs, a service of the Purdue University Libraries. Please contact epubs@purdue.edu for additional information. 


\title{
The low frequency performance of metamaterial barriers based on cellular structures
}

\author{
Srinivas Varanasi ${ }^{a, *}$, J. Stuart Bolton ${ }^{\mathrm{b}}$, Thomas H. Siegmund ${ }^{\mathrm{a}}$, Raymond \\ J. Cipra $^{a}$ \\ ${ }^{a}$ School of Mechanical Engineering, 585 Purdue Mall, West Lafayette, IN 47907-2088, \\ Phone: (765) 494-9766, U.S.A. \\ ${ }^{b}$ Ray W. Herrick Laboratories, School of Mechanical Engineering, Purdue University, \\ 140 S. Martin Jischke Drive, West Lafayette, IN 47907-2031, Phone: (765) 494-2139, \\ U.S.A.
}

\begin{abstract}
The high sound transmission loss (STL) metamaterials that have been suggested to-date commonly require the introduction of relatively heavy resonating or constraining components which limit the applicability of these solutions in typical noise control applications where it is desirable to minimize the treatment mass at a given STL. Here it is proposed that a panel consisting of an array of cellular unit structures can possess a high STL within a specified low frequency range without an undue mass penalty. The cellular acoustical metamaterial considered comprises a periodic arrangement of unit cells consisting of plates held in a grid-like frame (which itself is unsupported). It is suggested here that such a cellular panel can yield enhanced STL if the unit cell mass is apportioned appropriately between the unit cell plate and the surrounding grid-like frame, the effect being similar to the high STL observed in the low frequency range for a plate clamped along its edges. A finite element-model of a single unit cell was used to predict the normal incidence transmission loss of the periodic array by im-


posing boundary conditions that enforce spatial periodicity. Two material designs were compared: one in which the densities of the materials used for the unit plate panel and the grid were changed relative to each other, and a second where the relative thicknesses of the unit plate panel and the grid frame were varied. The numerical simulations indicate that benefits can be achieved in either case. However, the design based on density variations appears to be more effective than the approach based on geometry alteration in creating a relatively broad range of low frequency transmission loss enhancement.

Keywords: metamaterials, transmission loss, low frequency

\section{Introduction}

The design of lightweight passive treatments for noise barrier applications in the low frequency range has been a challenge due to the high mass per unit area required by mass-law-based designs. The blocking of low frequency sound can conventionally be achieved only by using relatively high areal masses, since stiffness-based or dissipation-based solutions are usually ineffective in that frequency range for unsupported, homogeneous panels [1]

Various alternative concepts have been proposed to address this problem. The idea of photonic band gaps was extended to acoustic waves, creating the concept of sonic band gaps. By allowing for periodic density variations,

\footnotetext{
${ }^{*}$ Corresponding author

Email addresses: svaranas@purdue.edu (Srinivas Varanasi), bolton@purdue .edu (J. Stuart Bolton), siegmund@purdue.edu (Thomas H. Siegmund), cipra@purdue .edu (Raymond J. Cipra)

Preprint submitted to Applied Acoustics

July 7, 2012
} 
acoustic wave propagation in some frequency bands can be forbidden [2]. But, the scale of the spatial periodicity of the structure in the direction of wave propagation needs to be on the order of the target wavelength, thus making this approach infeasible for shielding environmental noise when space is limited $[3,4]$.

Locally Resonant Sonic Materials (LRSMs) were proposed as a viable alternative for low frequency applications by Liu et al. [3, 5]. These authors studied a structure consisting of a slab of epoxy material with embedded, soft-coated lead balls acting as resonators. Specifically, two contrasting arrangements of resonators were considered: randomly dispersed resonators and a periodic lattice arrangement of resonators. In both these cases, the authors observed reduction in the measured transmission at certain frequencies in the low frequency range. The frequencies of reduced transmission corresponded to values calculated using multiple scattering theory. The high STL was attributed to negative effective elastic constants that resulted in the partially developed spectral gaps. Since both the periodic and random microstructures exhibited similar behavior, it was argued that the improvement is not dependent on the spatial periodicity of the resonator inclusions as long as the material is isotropic on the scale of the incident acoustic wavelength.

Subsequently, the concept of LRSMs has been investigated by several research groups. These studies consider LRSMs of two different types: (1) a matrix slab with embedded resonators as in the study conducted by Liu et al. $[3,5]$, and (2) a membrane with attached resonating masses supported 
and divided into a spatially periodic lattice of unit cells by a rigid grid.

Wester et al. [6] considered disks of polyester with embedded, coated steel balls acting as resonator elements and conducted transmission experiments using a standing wave tube $[7,8]$. By using a spring-mass-damper model, these authors reproduced experimentally observed qualitative characteristics in the STL. This model was further used to infer the cause for the high STL at certain frequencies. The peak in STL was observed to occur at an anti-resonance frequency that fell in between two resonance frequencies of the structure.

Yang et al. [9] considered a membrane structure with attached resonating masses and added a rigid grid to support the membrane. These authors reported beneficial results similar to those reported in refs. [3] and [5]. Similar results for tensioned membranes with and without finite permeability were reported earlier by Song and Bolton [10, 11]. Naify et al. [12] investigated the effect of membrane tension and the mass of the resonating elements on the STL characteristics of membrane-type materials supported by a rigid grid. Experimental results were compared to finite element simulations performed using coupled acoustic-structural analysis.

In LRSMs, the frequency intervals of high STL tend to be very narrow. In this context, to achieve high STLs over a wider frequency range, Yang et al. [13] considered stacking multiple LRSMs, each with different antiresonance frequencies. Once again, an important aspect of the structures they considered was the presence of a rigid grid around each unit cell.

Analytical studies have been performed to investigate the mechanism 
of sub-wavelength band gaps in LRSMs [14-16], and have linked the observed behavior to negative effective material parameters following from the particular microstructure of the mass-in-mass units used in LRSMs. Transmission dips are associated with zero space-averaged displacements that coincide with the occurrence of negative effective material parameters in membrane-based materials $[9,13]$. Similar features in the STL characteristics of tensioned, flexible circular membranes in a rigid wall were described theoretically in [17] and experimentally in [10], and the shape taken by the membrane at the STL peak frequencies is such that no energy can be radiated to infinity.

The spacing between embedded masses or the membrane support grid provide a length-scale to the problem, and thus, a frequency-scale. In this context, Lee et al.'s work [18] on periodically stiffened panels indicated that the STL at low frequencies in the realistic case of structures having a finite mass per unit area is dependent on the stiffness of the stiffeners and their mass relative to the panels. In the same way, it was also noted that the low frequency STL characteristics of a finite panel or pieces of poro-elastic media constrained at their edges are controlled by the panel's peripheral boundary conditions, their flexural stiffness and the mass of the supporting structure in the low frequency range [19-21]. In [21], the effect of the mass of the constraining grid elements on low frequency STL was considered in detail.

By uniting the ideas of the benefit of an edge constraint from a structural acoustics perspective, and the benefit of the microstructure of LRSMs in 
achieving higher STL, it is proposed that an infinitely extended microstructured panel (Fig. 1) with its unit cells having regions of heterogeneous material distribution or different geometries could serve as a novel acoustical metamaterial for barrier applications. While concepts in structural acoustics serve as the basis for this proposed material, its characteristic negative effective mass allows it to be classified as a LRSM [3].

The hypothesis underlying this study is that the cellular panel can approach two limiting configurations: that of the edge-constrained plate, and the limp panel. The edge-constraint limit would be achieved by indefinitely increasing the mass and stiffness of the frame in the unit cell, while the limp panel limit would be achieved by reducing the mass of the frame to zero (see Fig. 1 for the regions in the unit cell referred to as frame and plate). It is well known [19] that an edge-constrained plate has a high STL at very low frequencies due to the influence of the boundary condition combined with the flexural stiffness of the plate. Thus the question arises as to whether such a benefit could be realized without an undue mass penalty by controlling the frame properties in the unit cell? This question lead to the proposition that a specified areal mass (i.e., the averaged mass per unit area) could be distributed between the frame and plate in the proposed cellular panels to obtain an efficient acoustical metamaterial. The proposed design approach is called mass apportioning and its performance was assessed by comparing a set of cases that were areal mass neutral.

The work is presented in the following way. In the Materials section, the idea of cellular panels is introduced and the mass apportioning-based 
design approaches are explained. In the Methods section, the tools used for the analysis are described. In the Results section, the results supporting the hypothesis are presented, and later the results from the mass apportioning approach are shown to illustrate the high efficiency characteristics of cellular panels. Finally, these results are discussed from different perspectives such as the negative effective material parameters, their relation to LRSMs, etc., and the mechanisms behind the results are described.

\section{Materials}

\subsection{A Planar Plate-Based Cellular Metamaterial}

An acoustical metamaterial is proposed in the form of a cellular panel which extends infinitely in its plane (Fig. 1). The microstructure of the cellular panel consists of square unit cells, each composed of a thin plate and a supporting frame. The plate and the frame can be made of different materials. The normal-incidence STL characteristics of the infinitely extended cellular panel are determined by the geometry and material parameters of a single unit cell because of the spatial periodicity of the problem for normal incidence sound waves. Figure 2 shows the normal view of the overall panel together with a detail of the unit cell. When a normally incident plane wave excites the panel, the edges of the unit cell are subject to periodic boundary conditions, which were enforced by defining zero in-plane displacements.

The STL characteristics of the unit cell and thus the metamaterial panel, depend on its geometrical and material properties: i.e.,

$$
\mathrm{STL}=f\left(\rho_{p}, E_{p}, t_{p}, L_{p}, \rho_{f}, E_{f}, t_{f}, W_{f}\right)
$$


where the plate, i.e., the unit cell interior, has the density $\rho_{p}$, the elastic modulus $E_{p}$, the thickness $t_{p}$, and the length scale $L_{p}$. The frame, i.e., the cell wall surrounding the plate in the unit cell, possesses a density $\rho_{f}$, an elastic modulus $E_{f}$, a thickness $t_{f}$, and a width $W_{f}$.

\subsection{Material Design by Mass Apportioning}

Mass apportioning refers to the distribution of a given total unit cell mass, $m_{0}$, between the frame and plate in the unit cell while holding $m_{0}$ constant. In the present instance, mass apportioning was achieved in two ways: (1) by geometry-based apportioning, where the volumes of the plate and frame are adjusted while holding the material properties of the unit cell $\left(\rho_{p}, E_{p}, \rho_{f}, E_{f}\right)$ constant; or, (2) by material-based apportioning, where the material properties of the plate and frame are varied while holding the geometrical parameters of the unit cell $\left(t_{p}, L_{p}, t_{f}, W_{f}\right)$ constant. The contrast in the properties of the frame and the plate are quantified by using the mass ratio of the frame and the plate $\left(\mu=\rho_{f} V_{f}\right) /\left(\rho_{p} V_{p}\right)$ and the modulus ratio of the frame and the plate $\left(\eta=E_{f} / E_{p}\right)$. Here, $V_{f}$ is the volume of the frame, $\left(A_{f} t_{f}\right)$, where $A_{f}$ is the area of the frame given by $A_{f}=\left(L_{p}+W_{f}\right)^{2}-L_{p}^{2}$ and $V_{p}$ is the volume of the plate $\left(A_{p} t_{p}\right)$ and where $A_{p}$ is the area of the plate given by $A_{p}=L_{p}^{2}$ in the unit cell. The reference unit cell from which the designs for either approach were generated is defined by material properties $E_{r e f}$ and $\rho_{\text {ref }}$ where $E_{\text {ref }}$ is the elastic modulus and $\rho_{\text {ref }}$ is the material density for both the frame and the plate in the unit cell. Its geometric parameters, i.e., $t_{p}, t_{f}, L_{p}$ and $W_{f}$, are respectively defined as $t_{p}^{r e f}, t_{f}^{r e f}, L_{p}^{r e f}$ and $W_{f}^{r e f}$. The geometry-based mass apportioning was 
realized by varying the volumes of the frame and the plate through variation of thicknesses of the plate, $t_{p}$, and the frame, $t_{f}$, while holding $L_{p}$ and $W_{f}$ constant. Since the material properties are not varied, $\eta=1$ in all the geometry-based mass apportioning cases. The relation between the change in plate and frame thicknesses when holding the unit cell mass constant in order to obtain varying levels of $\mu$ (the mass contrast) is

$$
A_{f} \Delta t_{f}=-A_{p} \Delta t_{p}
$$

where $\Delta t_{f}$ and $\Delta t_{p}$ refer to the change in frame and plate thicknesses, respectively. The parameters $A_{f}, A_{p}, t_{f}$ and $t_{p}$ correspond to the frame area, plate area, thickness of the frame, and thickness of the plate, respectively, as defined before. Equation 2 can be used to generate a family of areal massneutral cases starting from the reference unit cell. For instance, the reference unit cell has a mass contrast $\mu$ equal to $A_{f}^{r e f} t_{f}^{r e f} / A_{p}^{r e f} t_{p}^{r e f}$ and the generated design has a mass contrast $\mu$ equal to $A_{f}^{r e f}\left(t_{f}^{r e f}+\Delta t_{f}\right) / A_{p}^{r e f}\left(t_{p}^{r e f}+\Delta t_{p}\right)$.

In material-based mass apportioning, the material properties of the plate and the frame were varied to obtain different levels of contrast ( $\mu$ and $\eta$ ) in the unit cell while holding its total mass the same as the reference unit cell. The values of $\rho_{f}$ and $\rho_{p}$ were determined by choosing the required value of $\mu$ and applying the constraint that the total mass of the unit cell remains constant. With increase in $\mu, \rho_{f}$ increases and $\rho_{p}$ decreases. At the same time, the modulus ratio $(\eta)$ was increased in proportion to $\mu$ by increasing the modulus of the frame $\left(E_{f}\right)$ while holding the modulus of the plate $\left(E_{p}\right)$ at the reference unit cell value. When $E_{f}$ is increased in proportion to $\mu$, the resonance frequencies associated with significant frame flexure were shifted 
to the higher frequencies, thereby allowing only for the plate flexure in the frequency range of interest $(0-2400 \mathrm{~Hz})$. The equations used to evaluate $\rho_{f}, \rho_{p}$, and $E_{f}$, respectively, for a chosen value of $\mu$ are:

$$
\begin{aligned}
& \rho_{f} V_{f}+\rho_{p} V_{p}=\rho_{r e f}\left(V_{f}+V_{p}\right), \\
& E_{f}=\left(\frac{\rho_{f}}{\rho_{p}}\right) E_{r e f} .
\end{aligned}
$$

Without loss of generality, for the numerical investigations, $E_{\text {ref }}$ and $\rho_{\text {ref }}$ were taken to be equal to $2 \mathrm{GPa}$ and $926.0 \mathrm{~kg} / \mathrm{m}^{3}$, respectively. The

reference unit cell geometric dimensions $t_{p}^{r e f}, t_{f}^{r e f}, L_{p}^{r e f}$ and $W_{f}^{r e f}$ were taken to be equal to $2.0 \mathrm{~mm}, 10.0 \mathrm{~mm}, 63.5 \mathrm{~mm}$, and $10.0 \mathrm{~mm}$, respectively. The areal mass for the chosen unit cell was $5 \mathrm{~kg} / \mathrm{m}^{2}$, and the volume of the frame in the reference unit cell was 3.65 times the volume of the plate. The choice of the geometric parameters $L_{p}^{r e f}$ and $W_{f}^{r e f}$ was motivated by the crosssectional dimensions of the Standing Wave Tube available (see Appendix A for the tube dimensions and for additional experimental verification of the acoustical analysis capabilities of the FE code). The material properties correspond to typical values for ABS plastic. The areal mass of this unit cell is a typical value for barrier materials used for noise control applications [19].

\section{Methods}

\subsection{Finite element model}

A three-dimensional finite element (FE) model was developed to study the acoustical properties of the unit cell representing the proposed cellular LRSM. The ABAQUS FE code was employed [22]. The model was built to 
reproduce the standing wave tube setup used for characterizing barrier materials [8] (see Fig. 3). The numerical analysis was based on a steady-state, dynamically-coupled structural-acoustic procedure that falls into the broad category of linear perturbation methods [22]. A numerical-experimental verification of the acoustical analysis was performed and is described in Appendix A. The model consisted of two acoustic domains (upstream and downstream) and a solid domain consisting of a unit cell separating the two acoustic domains. The acoustic domains in the model were given a square cross-section with dimensions of $L_{p}$ by $L_{p}$. In order to simplify the analysis for the parametric study component of the paper, the frame of the unit cell was not exposed to the acoustic loading. The lengths of the up- and downstream acoustic domains were taken to be $500.0 \mathrm{~mm}$ each. Acoustical elements (8-node linear hexahedral) were used to discretize the acoustical domains, while structural elements (20-node quadratic hexahedral, reduced integration) were used to discretize the solid domain. The element size in the acoustic domain was chosen to conform to the rule that there should be more than six elements per wavelength at the highest frequency in the range considered. In the unit cell, the element size was so chosen to capture the flexural behavior as well as the dynamic behavior. A minimum of three layers of elements were used through the thickness $\left(t_{p}\right)$ of the plate to satisfy the former requirement, and the element sizes were also chosen so that there were at least six elements in one shear wavelength at the highest frequency considered to ensure the latter. For example, a typical element size in the duct was $10.0 \mathrm{~mm}$ (in the wave propagation direction) which 
ensures that there were 11 elements per wavelength at $3000 \mathrm{~Hz}$. The inplane element size in the unit cell was $2.0 \mathrm{~mm}$ (the shear wave speed for the reference material properties mentioned in Sec. 2.2 is roughly $900 \mathrm{~m} / \mathrm{s}$ ) which meant that there were 150 elements per wavelength at $3000 \mathrm{~Hz}$; and there were three layers of elements through the thickness of the panel. Mesh convergence studies were performed on the model and showed satisfactory accuracy at this level of discretization (see Appendix B for details).

A sound source radiating a single frequency sound pressure was located at the upstream end of the acoustic domain while the downstream end of the acoustic domain was given an anechoic termination by imposing a specific acoustic impedance of $\rho_{0} c$ : i.e., the characteristic impedance of air. Here $\rho_{0}$ is the density of air and $c$ is the speed of sound in air. The model was executed one-frequency-at-a-time with a frequency interval of $8 \mathrm{~Hz}$ to cover a specified frequency $(0-2400 \mathrm{~Hz})$ range with a unit amplitude input.

To evaluate the characteristics of the unit cell, predicted sound pressures were recorded by virtual "microphones" at two axial locations in both the upstream and downstream acoustic domains at each frequency within the specified range $(0-2400 \mathrm{~Hz})$. The transfer functions between the first microphone and the other three microphones were determined using the single load method and were then used to evaluate the STL characteristics of the unit cell $[23,24]$. The pressure measurement locations were chosen so that they were at least a distance $L_{p}$ away from the unit cell on either side. The independence of the results on the choice of pressure measurement locations was confirmed by processing data obtained from different sets of 
measurement pairs for the same model.

The unit cell FE model described above was also used to conduct modal analysis based on linear perturbation methods [22] to determine the eigenmodes of the unit cells. Unlike the above model, the latter model does not include the acoustic domains.

\section{Results}

\subsection{Verification of Underlying Hypothesis}

To verify that the unit cell behavior would converge to the edge-constrained plate limit, the mass of the frame of the unit cell was progressively increased, thereby increasing the mass contrast $(\mu)$, while holding all the geometric parameters of the unit cell and the material parameters of the plate of the unit cell the same as that of the reference unit cell. Also, the modulus of the frame $\left(E_{f}\right)$ was increased in proportion to $\mu$ so that the resonance frequencies associated with frame flexure remained at approximately their original frequencies, thereby allowing only for the plate flexure in the frequency range of interest $(0-2400 \mathrm{~Hz})$. Figure 4(a) shows the predicted STL obtained for increasing values of $\mu$ in the range of $0-10000$. These results were compared with the predicted STL for an edge-constrained plate, whose geometry and material specifications corresponded to those of the plate in the reference unit cell. Figure 4(a), shows the predicted STLs and Fig. 4(b) shows the values of the peak and dip frequencies for the STL as shown in Fig. 4(a). The dip frequency converges to $1272 \mathrm{~Hz}$ which is also the dip frequency of the edge-constrained plate. Similarly, the location of 
the peak STL converges to $0 \mathrm{~Hz}$ as in the edge-constrained plate. The dip frequencies can be compared with the first flexural resonance frequency of the edge-constrained plate. By using the approximate expression for a fully clamped plate [25], i.e.,

$$
f_{c l 1}=\frac{\lambda \pi}{4 \sqrt{3} \sqrt{\left(1-\nu^{2}\right)}} \frac{t_{p}}{l_{p}^{2}} \sqrt{\frac{E_{p}}{\rho_{p}}}
$$

the resonance frequency was estimated to be $1292 \mathrm{~Hz}$, which is in good agreement with the prediction of the FE model. In Eq. $4, \lambda=3.66$, for a plate clamped on all four sides. All the other parameters are the same as defined for the plate in the unit cell given in Eq. 1.

On the other hand, the limp panel limit was verified by reducing the mass of the frame of the unit cell to zero while holding the material properties of the plate the same as in the reference unit cell. In Fig. 5, the FE-predicted STL is compared with the analytical result, i.e.,

$$
T=\frac{2 \rho_{0} c}{2 \rho_{0} c+j 2 \pi f m_{s}}
$$

for a limp panel. In Eq. 5, $T$ is the transmission coefficient, $f$ is the frequency in Hertz and $m_{s}$ is the areal mass of the unit cell. The STL is given by $-20 \log (\|T\|)$ where $\|T\|)$ is the absolute value of the amplitude transmission coefficient. A close agreement between the simulation result and the mass law was obtained.

These results confirm that the unit cell behavior in the limit possesses the characteristics of either a clamped plate or a limp panel. Still, the cases considered so far are not mass neutral. Since the condition of mass 
neutrality is a relevant practical constraint, the next subsection explores material designs in which no mass is added.

\subsection{Mass apportioning}

A series of material designs were generated for the geometry-based and material-based approaches by using Eqs. 2 and 3, respectively: the corresponding unit cell properties are listed in Tables 1 and 2. Figures 6 and 7 show the predicted STL characteristics for the geometry-based and material-based approaches, respectively, for different values of $\mu$. As stated before, all unit cells considered for the two approaches possessed the same areal mass as the reference unit cell: i.e., $5 \mathrm{~kg} / \mathrm{m}^{2}$. Figure 8 compares the predicted STLs from both approaches for the same mass contrast value $(\mu)$. For each unit cell, its first flexural eigenmode $\left(f_{b 1}\right)$ was determined by modal analysis, and the predicted frequencies were compared with the resonance frequency of a fully clamped plate $\left(f_{c l 1}\right)$ having the same material and geometric specifications as the unit cell interior. The frequency, $f_{c l 1}$ was evaluated using the Eq. 4: Tables 3 and 4 list $f_{b 1}$ and $f_{c l 1}$.

From Figs. 6 and 7 it can be seen that the STL characteristics for all cases share common features. The predicted STL starts from a zero value at $0 \mathrm{~Hz}$, next peaks, and then reaches a dip having a zero STL. Higher frequency peaks and dips occur at varying frequency intervals. This trend is less distinctly seen for low values of $\mu$, say $\mu=0.1$, as the peak and dip occur very close to each other. When focussing on the region from $0 \mathrm{~Hz}$ to the dip frequency in each case, there is a region of benefit in terms of high STL around the peak. The relative location of the frequency at the STL 
peak in between $0 \mathrm{~Hz}$ and the dip frequency also varies. The dip frequency in the predicted STL corresponds to the first eigenmode of the unit cell $\left(f_{b 1}\right)$ characterized predominantly by bending deformation of the plate. By comparing $f_{b 1}$ to $f_{c l 1}$, it can be seen that the two characteristic frequencies are in close proximity, and that the difference between these normalized by $f_{c l 1}$ decreases with increasing value of $(\mu)$ in both the approaches (see Tables 3 and 4). This indicates that the first dip is predominantly determined by $f_{c l 1}$ of the plate in the unit cell which is in turn determined by its material and geometric specifications, and can be used in marking the dip frequency for any unit cell. By using Eq. 4, it can be seen that $f_{c l 1}$ is proportional to $t_{p} / l_{p}^{2} \sqrt{E_{p} / \rho_{p}}$. On the other hand, the zero STL at $0 \mathrm{~Hz}$ is due to the unconstrained displacement normal to the unit cell, and is consistent with Eq. 5 when $f$ approaches $0 \mathrm{~Hz}$.

From Figs. 6 and 7, it can be seen that irrespective of the mass apportioning approach used, the cellular panels offer beneficial STL characteristics in the low frequency region when compared to the characteristic limp panels when maintaining mass neutrality. Specifically, for material-based designs (see Fig. 7), it can be seen that as $\mu$ is increased, the high STL region broadens in the low frequency range while the peak STL occurs at progressively higher frequencies with the predicted STL almost converging when $\mu$ reaches a value on the $\mathrm{O}(100)$. At the same time, the frequency span between the peak and the dip increases. Thus, it can be inferred that a microstructure design with a unit cell possessing a relatively heavy and stiff frame combined with a relatively light plate-type cell interior performs 
better in low frequency applications than a simple limp panel having the same areal mass. On the other hand, in the case of the geometry-based designs (see Fig. 6), it can be seen that the region of benefit shrinks and moves towards a lower frequency as $\mu$ increases. Finally, from Fig. 8, it can be seen that the material-based mass apportioning approach gives better results than the geometry-based approach, if the objective is to maximize the span of the benefit region. If instead, the objective were to maximize the STL over a narrow band in a particular very low frequency range, for a given areal mass and length-scale, the geometry-based approach would provide a better solution.

By analyzing the expression for $f_{c l 1}$ (Eq. 4) as the locator of the first dip in the predicted STL, it can be seen that $L_{p}$ plays the primary role among all the parameters involved in determining the location of the dip due to its inverse square relationship with $f_{c l 1}$, essentially determining the frequency range of operation. The other three parameters, namely, $E_{p}, \rho_{p}$, and $t_{p}$ are of secondary influence. In geometry-based apportioning, $t_{p}$ was decreased to increase the level of mass contrast in the unit cell, $\mu$, by keeping $E_{p}$ and $\rho_{p}$ constant. On the other hand, in material-based mass apportioning, $\rho_{p}$ was decreased while keeping the other two parameters constant in order to increase $\mu$. Since $f_{c l 1}$ (see Eq. 4) is directly proportional to $t_{p}$, and inversely proportional to $\sqrt{\rho}$, the two approaches move the dip in opposite directions for increasing $\mu$, and therefore serve as two complementary design approaches. Irrespective of the location of the dip, it can further be seen from Tables 3 and 4 , that the relative location of the peak, $\left(f_{d i p}-f_{\text {peak }}\right) / f_{d i p}$, 
increases with increasing value of $\mu$ within the mass neutral cases.

While in the cases presented above $E_{f}$ was varied in proportion to $\mu$, Fig. 9 shows the effect of varying $E_{p}$ or $E_{f}$ independently while holding the other properties of a unit cell constant and Table 5 lists the cases. The starting unit cell chosen for this study was case 3 in Table 2 . When considering the cases having constant $\mu$ but varying $E_{f}$ or $E_{p}$, it can be seen that both the peak and dip locations are dependent on the two moduli $\left(E_{f}, E_{p}\right)$ but with varying sensitivity. While the relative location of the peaks, $\left(f_{\text {dip }}-f_{\text {peak }}\right) / f_{\text {dip }}$, remain nearly constant (Table 5$)$, their absolute locations are more sensitive to $E_{p}$ than $E_{f}$.

\subsection{Effect of exposing the frame to acoustic loading}

All results presented so far are based on a simplified unit cell model where the frame was not exposed to acoustic loading. To study the effect of acoustic loading on the frame, the material-based mass apportioning cases presented above were investigated after exposing their frames to the incident sound field. Figure 10 shows the predicted STL with and without exposing the frame to acoustic loading for the cases $\mu=0.1,1,10$ and 100, respectively. The ratio of the frame to the plate areas $\left(A_{f} / A_{p}\right)$ was assumed to be 0.73 and 0.34 , respectively, such that the values for $t_{f}$ were $10.00 \mathrm{~mm}$ and $21.49 \mathrm{~mm}$.

By comparing the predicted STL data for simulations with and without the frame being exposed to acoustic loading, the location of the dip in the predicted STL does not change but the peak shifts to a higher frequency. Since the location of the dip is determined by $f_{b 1}$, and since this frequency is 
independent of whether the frame is exposed to the acoustic loading or not, the dip location is not affected by frame exposure. On the other hand the occurrence of the peak is characterized by a combination of translational motion of the frame and the flexural bending of the plate: it is thus affected by the frame being exposed to the sound field. Nonetheless, the qualitative features of the predicted STL remain the same in either case for all levels of mass contrast $(\mu)$. By comparing the predicted STLs of the cases with different values of $A_{f}$, and the frames being exposed to acoustic load, it can be seen that the shift of the peak to higher frequencies can be reduced by reducing the frame area.

\section{Discussion}

Acoustical metamaterials $[3,9]$ can be characterized as possessing negative effective material parameters. To obtain the effective parameters, an equivalent homogenized material model is assumed and predicted (or measured) sound transmission characteristics are projected onto this reference model such that the effective material properties for the selected homogeneous model are obtained. For instance, Liu et. al. [3] used the amplitude transmission coefficient expression for a slab of homogeneous material at normal incidence as the reference model. The averaged mass density of the composite was assumed to be the density of the homogenized material, and thus the effective modulus could be obtained. Yang et al. [9] obtained the effective dynamic mass of membrane-based materials by dividing the averaged stress by the average acceleration. Fokin et al. [26] suggested using 
both the reflection and transmission coefficients in obtaining the effective parameters.

In the present work, the limp panel was assumed to serve as the homogenized structure for comparison with the cellular panel, and its effective areal mass was obtained by inverting the predicted STL using the expression for the sound transmission as given in Eq. 5. The expression for the effective dynamic areal mass after the inversion is then

$$
m_{s}(f)=\frac{2 \rho_{0} c\left(1-T_{p r}\right)}{j 2 \pi f T_{p r}} .
$$

In Eq. $6, m_{s}(f)$ is the effective areal mass as a function of frequency, $f$, in Hertz and $T_{p r}$ is the predicted transmission coefficient from the FE simulations. As one example, Eq. 6 was applied to calculate the effective mass for the case $(\mu=10)$ in the material-based approach. Figures 11(a) and (b) show the real and imaginary parts of the effective mass normalized by the averaged areal mass $m_{0} /\left(L_{p}+W_{f}\right)^{2}$ where $m_{0}$ is the total unit cell mass, and the other parameters are as defined in Eq. 1. The STL is also shown for reference. The magnitude of the effective mass is higher than the spaceaveraged mass in the frequency range of 0-900 Hz. It can also be seen that the effective mass is larger than the space-averaged mass by at least an order of magnitude in the frequency range of 700-900 Hz. It can also be seen that effective mass is strongly negative near the peak STL. This observation is in agreement with the negative mass effect discussed in the literature [9] and suggests that the mechanism behind the high STL is similar to that for the metamaterials proposed by the other researchers [9]. From Fig. 11(b), 
it can be seen that the imaginary part of the effective mass is at least an order of magnitude smaller than the real part, and except for the region near the peak STL, it is almost zero in magnitude.

To understand the mechanism behind the predicted high STL in particular frequency ranges, the relative displacement field amplitude of the unit cells was observed at the peak and dip frequency locations. Figure 12 shows the real component of the relative displacement field amplitude normal to the unit cell (in the sound propagation direction) for a material-based mass apportioning case with $\mu=10$ at the (a) peak (800 Hz) and (b) dip (1936 $\mathrm{Hz}$ ) frequencies, respectively. From Fig. 12(a), it can be seen that at the frequency of peak STL, the displacement associated with bending of the unit cell interior and the translational motion of the frame are out of phase. Particularly in the plate, it can be observed that its central region and the region around it were out of phase unlike in the case for the limp panel where the motion of all the points is in-phase. On the other hand, from Fig. 12(b), it can be seen that at the frequency of the minimum STL, deformation is confined to bending of the unit cell interior and that translational motion of the frame is absent. Such a displacement field is very much similar to what is observed in an edge-constrained plate first flexural resonance. These observations suggest that the relative out-of-phase motion observed at the peak STL effectively causes the unit cell to behave like a rigid wall (i.e., a surface having zero net volume velocity) thus resulting in the high STL.

The averaged phase of the complex displacement (phase in degrees when 
complex displacement is expressed in polar form) along the wave propagation direction over the unit cell interior was computed across the frequency range $0-2400 \mathrm{~Hz}$. Figure 13 shows the averaged phase and the predicted STL again for the case of $\mu=10$ and the mass-apportioning material design. It was observed that there is a switch in the phase from a negative to a positive value at the frequency of peak STL, which suggests that the net volume displacement amplitude approaches zero for high STL supporting the qualitative behavior discussed in connection with the results of Fig. 12.

\section{Conclusions}

In the present work it was proposed and demonstrated that a planar cellular metamaterial possessing a microstructure consisting of a heavy and stiff cell wall (i.e., a frame) surrounding a plate-like cell interior possesses significantly increased STL in a low frequency range compared to a homogeneous solid of equal areal mass in the form of a limp panel. Two design strategies based on the idea of mass redistribution, namely, material-based and geometry-based approaches, were studied. It was found that by using the material-based approach, it was possible to arrive at the optimal solution for a given length-scale of the unit cell, geometry and average areal mass. On the other hand, it was found that the geometry-based approach would be more helpful in finding solutions having very high sound transmission loss in relatively narrow low frequency bands compared to the material-based approach for the same unit cell length-scale and average areal mass. 


\section{Acknowledgements}

The authors gratefully acknowledge the financial support provided by the United States Air Force Office of Scientific Research through the grant FQ8671-090162. 


\section{List of Tables}

1 Geometry-based mass apportioning cases . . . . . . . . . . . 28

2 Material-based mass apportioning cases . . . . . . . . . . . . 28

3 Characteristic frequencies of geometry-based mass apportioning cases . . . . . . . . . . . . . . . . . . . . . . . 29

4 Characteristic frequencies of material-based mass apportioning cases . . . . . . . . . . . . . . . . . . . . 29

5 Effect of varying $E_{p}$ or $E_{f}$ on unit cell STL characteristics. . 30

\section{List of Figures}

1 Cellular panel with its unit cell, and a cutaway view of its unit cell defining its component dimensions. . . . . . . . . . 31

2 A normal view of the cellular panel illustrating the periodic boundary conditions on a unit cell. In the magnified view of a unit cell, the edges where the periodic boundary conditions (in-planar displacements $u 1$ and $u 2$ equal to zero) are imposed are marked as ABCD, and shown as dotted lines. . 32

3 A schematic drawing of the FE simulation model for characterizing cellular panels. Virtual microphones are labeled as M1, M2, M3, and M4 in the schematic. . . . . . . . . . 33 
4 (a) Predicted STL for the unit cell for a range of $\mu$ values, compared to the case of an edge-constrained plate of dimensions identical to the plate in the unit cell, and the predictions from Eq. 4. (b) Frequencies corresponding to the peak and dip STL values as a function of $\mu$. . . . . . . . . 34

5 The STL predicted by the FE analysis of the unit cell for the case of $\mu=0$ (mass of the frame is zero) compared to the STL calculated from the mass law (Eq. 5). . . . . . . . . 35

6 STL characteristics of cellular panels designed through geometrybased mass apportioning: comparison to the first flexural eigenfrequency - $f_{b 1}$, the first flexural mode frequency of a plate clamped on all four sides- $f_{c l 1}$ (Eq. 4), and the predicted STL for a mass law (Eq. 5) panel having an areal mass equal to the averaged areal mass of the unit cell $\left(m_{0} /\left(L_{p}+W_{f}\right)^{2}\right) . \quad 36$

$7 \quad$ STL characteristics of cellular panels designed through materialbased mass apportioning, comparison to the first flexural eigenfrequency - $f_{b 1}$, the first flexural mode frequency of a fully clamped plate- $f_{c l 1}$ (Eq. 4) for the cases of $\mu=0.1,1,10$, and the predicted STL for a mass law (Eq. 5) panel having an areal mass equal to the averaged areal mass of the unit cell $\left(m_{0} /\left(L_{p}+W_{f}\right)^{2}\right) \ldots \ldots \ldots \ldots \ldots$ 
8 Comparison of predicted STL for material-based and geometrybased design approaches for $\mu=8.29$ and constant unit cell mass $m_{0}$ equal to that of the reference unit cell. Comparison to STL obtained for a mass law panel having an areal mass equal to the averaged areal mass of the unit cell $m_{0} /\left(L_{p}+W_{f}\right)^{2} .38$

9 Effect of varying $E_{p}$ and $E_{f}$ on predicted STL while holding all the other material and geometric parameters of the unit cell constant ( $\mu=10$ in material-based mass apportioning design). $E_{p}^{0}$ and $E_{f}^{0}$ are the unit cell material properties (see Table 2). . . . . . . . . . . . . . . 39

10 Effect of exposing the frame in the unit cell to acoustic loading on predicted STL for cases (a) $\mu=0.1$, (b) $\mu=1$, (c) $\mu=10$, and (d) $\mu=100$ in material-based mass apportioning design. . . . . . . . . . . . . . . . 40

11 Real (a) and imaginary (b) parts of the effective mass (Eq. 6) as a function of frequency, and in comparison to the corresponding STL for the case of $\mu=10$ through materialbased mass apportioning. Both the components are normalized using the averaged areal mass of the unit cell $m_{a 0}=$ $m_{0} /\left(L_{p}+W_{f}\right)^{2}$ where $m_{0}$ is the total mass of the unit cell, and the other geometric parameters were defined in Eq. 1. . 41 
12 A qualitative comparison of predicted displacement in the $x$-direction (Re of $\operatorname{Re}+\jmath \operatorname{Im})$ at the (a) peak and (b) dip frequencies respectively of a unit cell in material-based approach with $\mu=10$. Red and blue indicate positive and negative displacements along the duct axis, respectively. . . 42

13 Averaged displacement phase for the case of $\mu=10$ in materialbased mass apportioning averaged over the plate region in the unit cell. . . . . . . . . . . . . . . . . . 43

A.1 (a) A schematic showing the standard Standing Wave Tube Setup [8] used for characterizing barrier materials, and (b) a schematic drawing illustrating the placement of test specimen in the standing wave tube set-up and the need for imposing the specified boundary conditions for experimental validation. 49

A.2 Comparison of predicted and measured STLs for an extruded acrylic test sample. . . . . . . . . . . . . . 50 50

B.1 Mesh convergence study for different element sizes in the solid domain of the simulation setup. . . . . . . . . 52 
Table 1: Geometry-based mass apportioning cases

\begin{tabular}{|l|l|l|}
\hline$\mu$ & $t_{p}[\mathrm{~mm}]$ & $t_{f}[\mathrm{~mm}]$ \\
\hline 3.65 & 2.00 & 10.000 \\
\hline 4.31 & 1.75 & 10.343 \\
\hline 5.19 & 1.50 & 10.690 \\
\hline 6.43 & 1.25 & 11.030 \\
\hline 8.29 & 1.00 & 11.370 \\
\hline
\end{tabular}

Table 2: Material-based mass apportioning cases

\begin{tabular}{|l|l|l|l|}
\hline$\mu$ & $\rho_{p}\left[\mathrm{~kg} / \mathrm{m}^{3}\right]$ & $\rho_{f}\left[\mathrm{~kg} / \mathrm{m}^{3}\right]$ & $E_{f}[\mathrm{GPa}]$ \\
\hline 0.1 & 3910.0 & 107.0 & 0.055 \\
\hline 1.0 & 2151.0 & 590.0 & 0.549 \\
\hline 10.0 & 391.0 & 1073.0 & 5.490 \\
\hline 100.0 & 43.0 & 1168.0 & 54.900 \\
\hline
\end{tabular}


Table 3: Characteristic frequencies of geometry-based mass apportioning cases

\begin{tabular}{|l|l|l|l|l|}
\hline$\mu$ & $f_{c l 1}[\mathrm{~Hz}]$ & $f_{b 1}=f_{\text {dip }}[\mathrm{Hz}]$ & $f_{\text {peak }}[\mathrm{Hz}]$ & $\left(f_{\text {dip }}-f_{\text {peak }}\right) / f_{\text {dip }}$ \\
\hline 3.65 & 1291.5 & 1255.4 & 760 & 0.394 \\
\hline 4.31 & 1130.0 & 1102.8 & 632 & 0.427 \\
\hline 5.19 & 968.6 & 949.5 & 512 & 0.46 \\
\hline 6.43 & 807.2 & 794.4 & 392 & 0.5 \\
\hline 8.29 & 645.7 & 637.4 & 280 & 0.56 \\
\hline
\end{tabular}

Table 4: Characteristic frequencies of material-based mass apportioning cases

\begin{tabular}{|l|l|l|}
\hline$\mu$ & $f_{c l 1}[\mathrm{~Hz}]$ & $f_{b 1}=f_{d i p}[\mathrm{~Hz}]$ \\
\hline 0.1 & 628.5 & 567.7 \\
\hline 1.0 & 847.4 & 816.0 \\
\hline 10.0 & 1987.4 & 1945.5 \\
\hline 100.0 & 5993.1 & 5943.1 \\
\hline
\end{tabular}


Table 5: Effect of varying $E_{p}$ or $E_{f}$ on unit cell STL characteristics.

\begin{tabular}{|l|l|l|l|}
\hline Case & $f_{\text {dip }}[\mathrm{Hz}]$ & $f_{\text {peak }}[\mathrm{Hz}]$ & $\left(f_{\text {dip }}-f_{\text {peak }}\right) / f_{\text {dip }}$ \\
\hline$E_{f 0}, E_{p 0}$ & 1936 & 792 & 0.59 \\
\hline$E_{f 0} / 10, E_{p 0}$ & 1648 & 648 & 0.60 \\
\hline $10 E_{f 0}, E_{p 0}$ & 1984 & 832 & 0.58 \\
\hline$E_{f 0}, E_{p 0} / 10$ & 624 & 264 & 0.58 \\
\hline
\end{tabular}



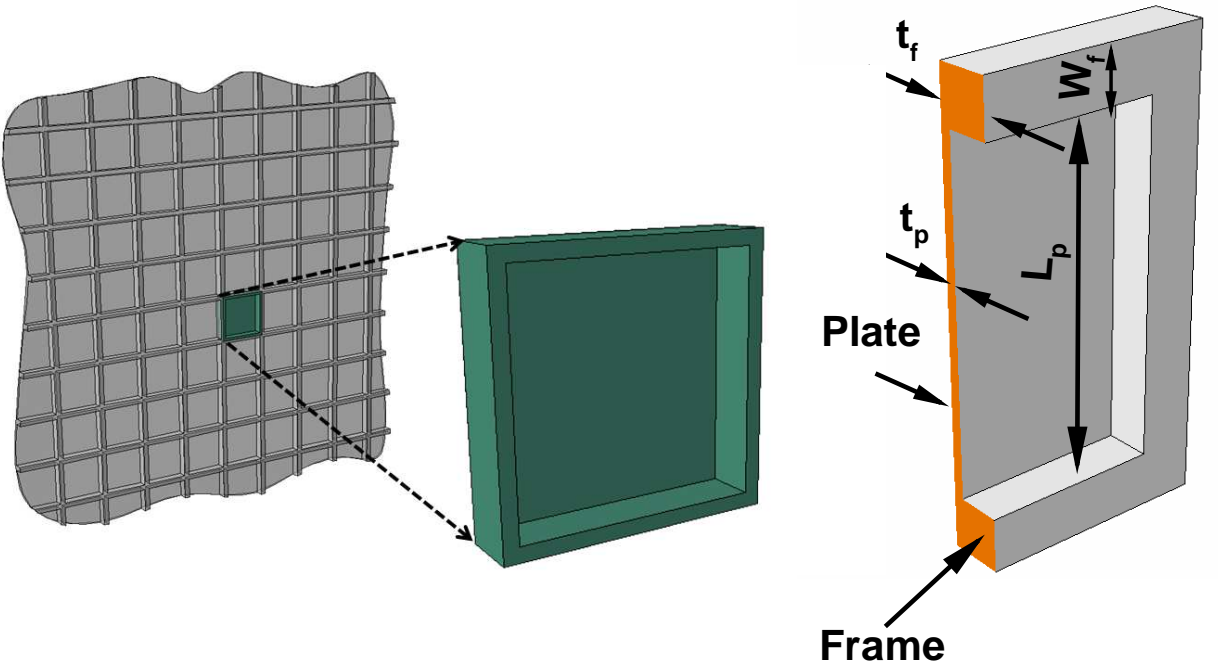

(a) (b)

Figure 1: Cellular panel with its unit cell, and a cutaway view of its unit cell defining its component dimensions. 


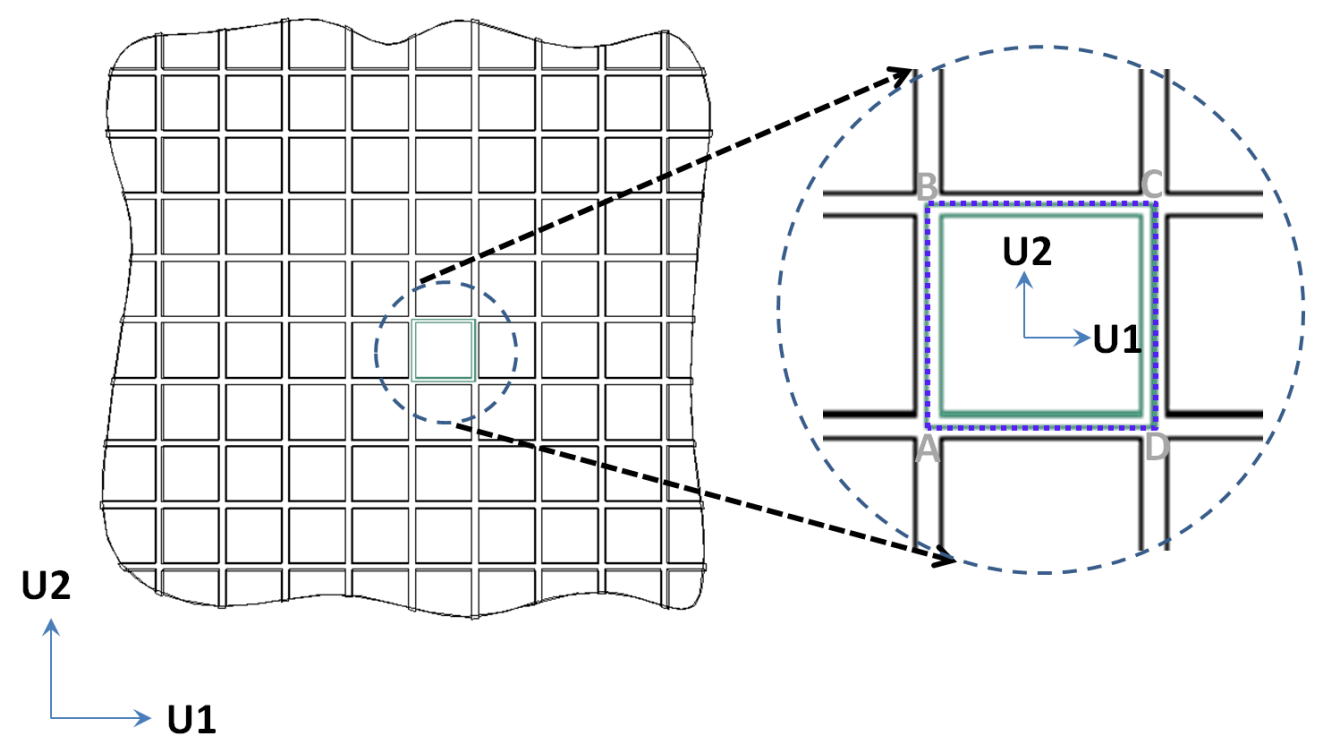

Figure 2: A normal view of the cellular panel illustrating the periodic boundary conditions on a unit cell. In the magnified view of a unit cell, the edges where the periodic boundary conditions (in-planar displacements $u 1$ and $u 2$ equal to zero) are imposed are marked as ABCD, and shown as dotted lines. 


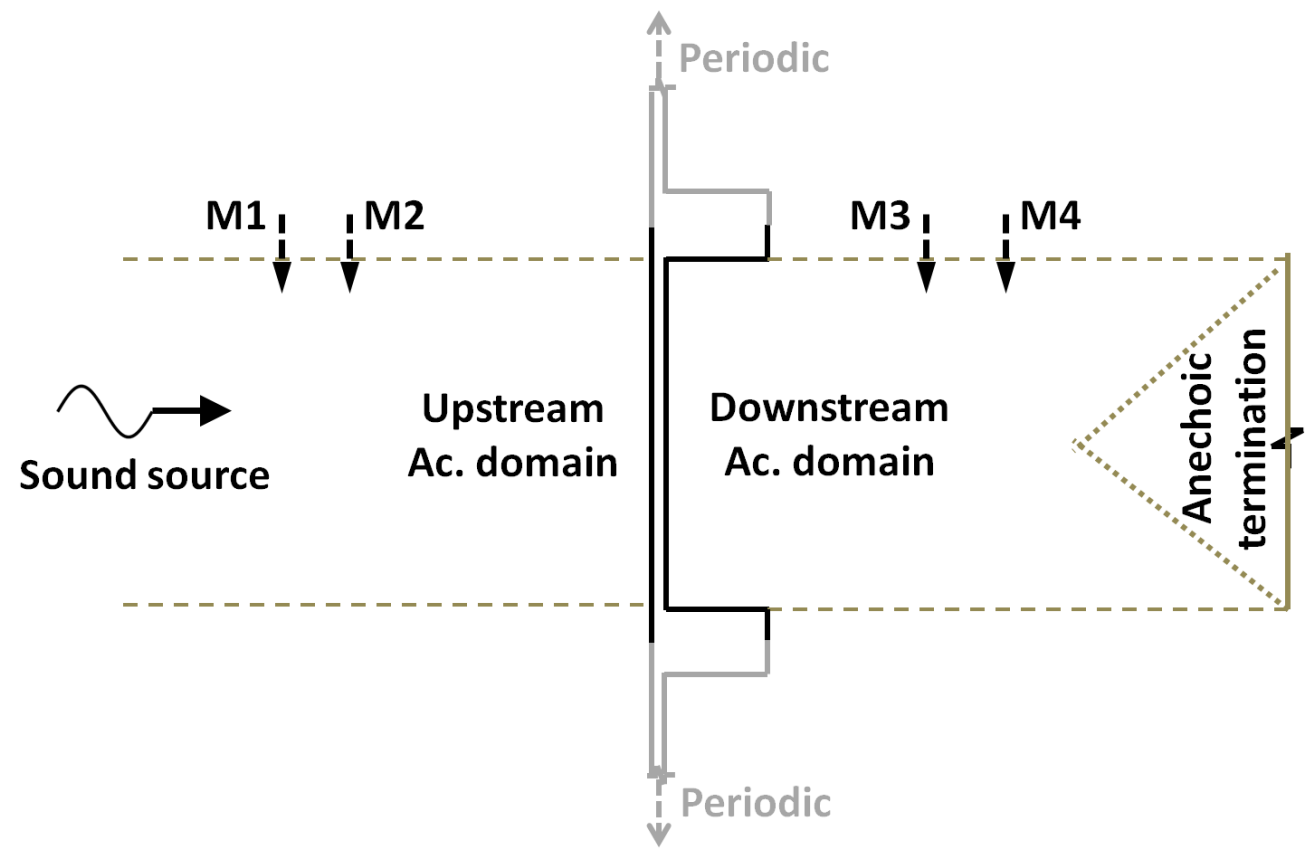

Cellular panel

Figure 3: A schematic drawing of the FE simulation model for characterizing cellular panels. Virtual microphones are labeled as M1, M2, M3, and M4 in the schematic. 


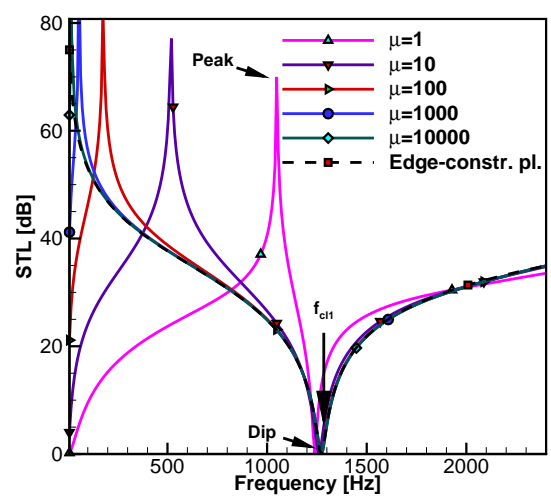

(a)

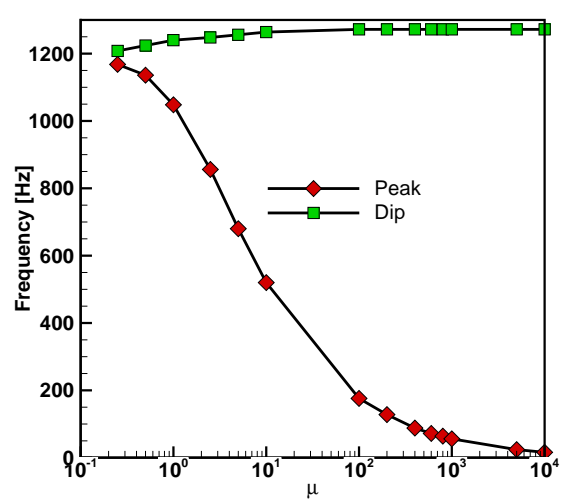

(b)

Figure 4: (a) Predicted STL for the unit cell for a range of $\mu$ values, compared to the case of an edge-constrained plate of dimensions identical to the plate in the unit cell, and the predictions from Eq. 4. (b) Frequencies corresponding to the peak and dip STL values as a function of $\mu$. 


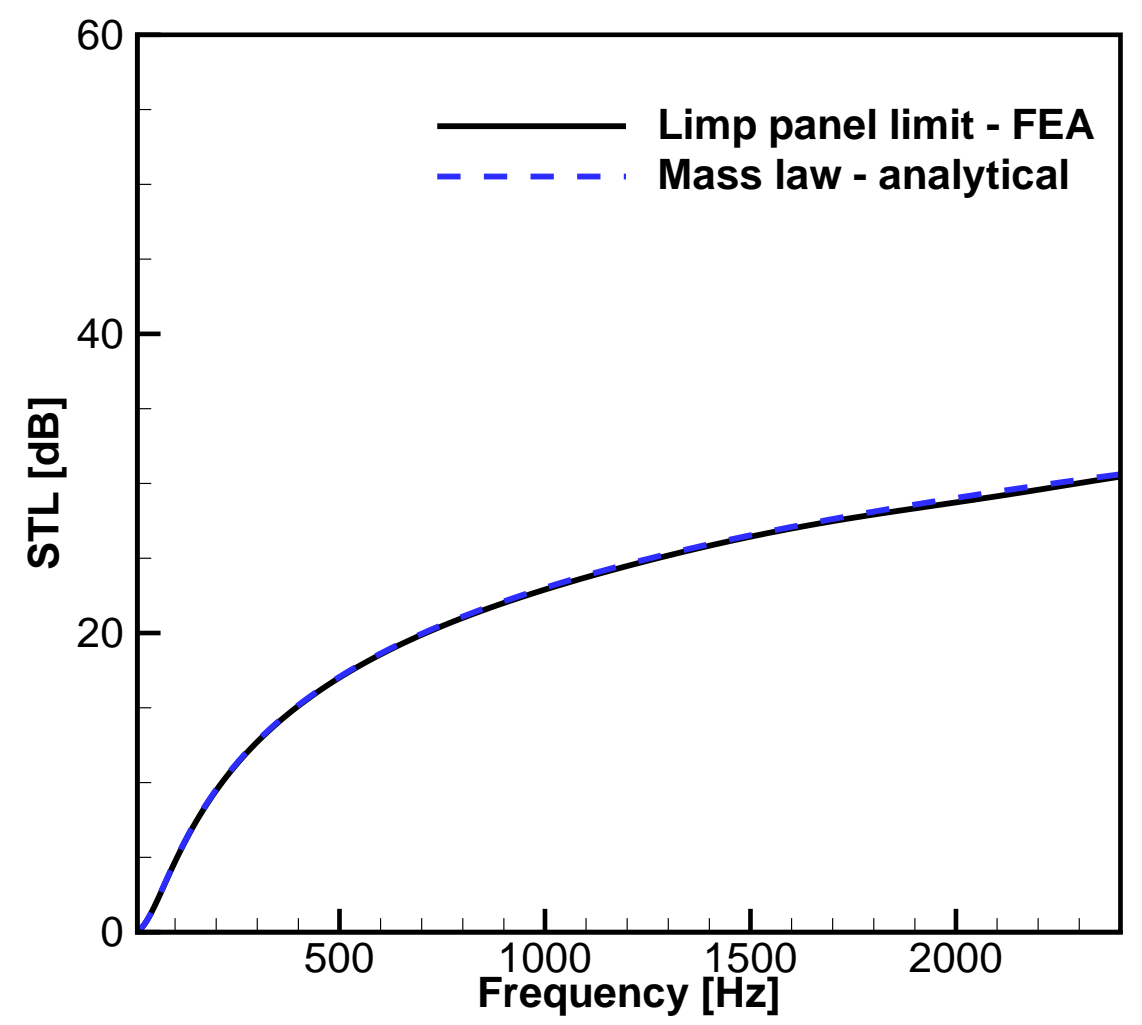

Figure 5: The STL predicted by the FE analysis of the unit cell for the case of $\mu=0$ (mass of the frame is zero) compared to the STL calculated from the mass law (Eq. 5). 


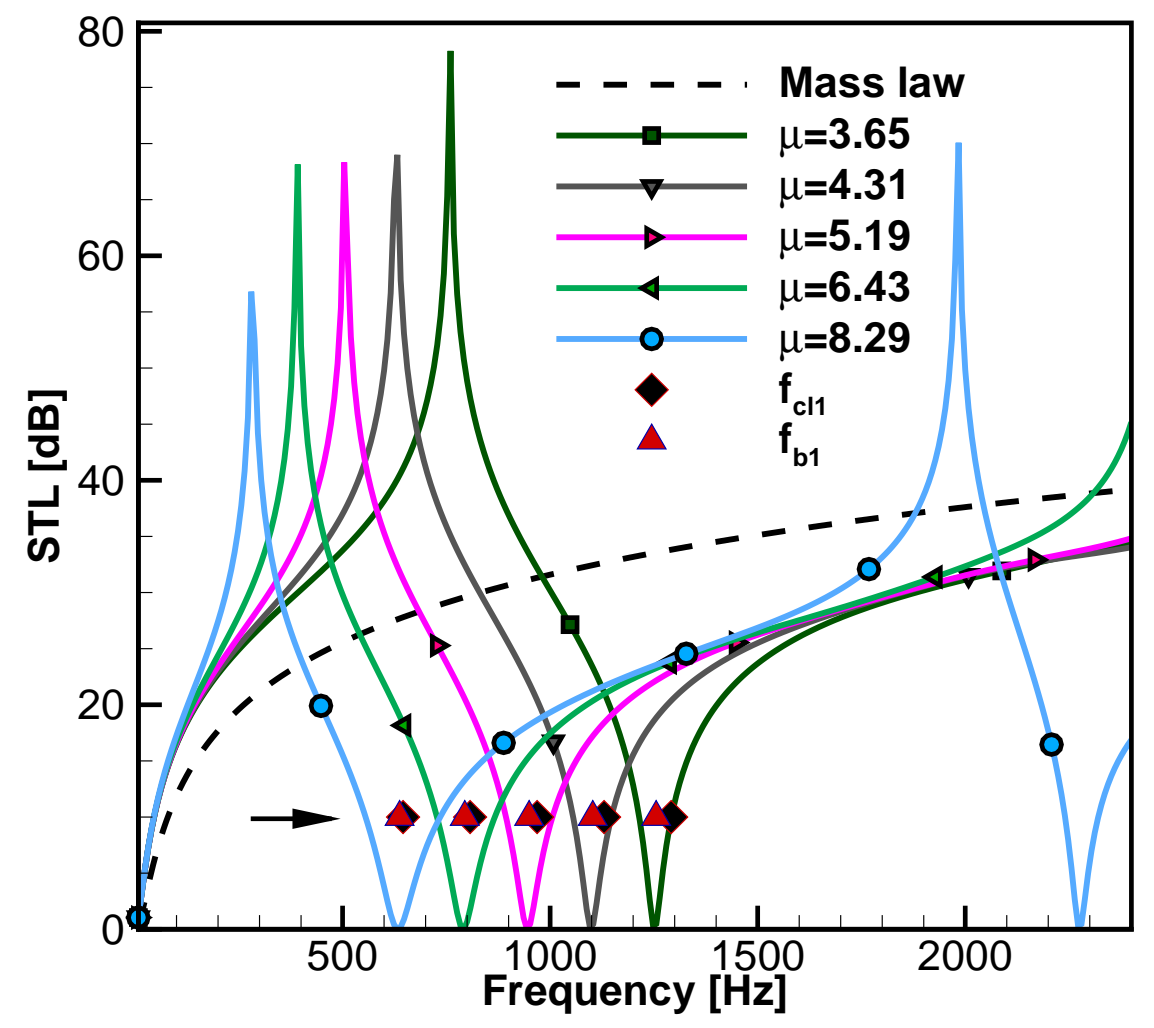

Figure 6: STL characteristics of cellular panels designed through geometrybased mass apportioning: comparison to the first flexural eigenfrequency $f_{b 1}$, the first flexural mode frequency of a plate clamped on all four sides- $f_{c l 1}$ (Eq. 4), and the predicted STL for a mass law (Eq. 5) panel having an areal mass equal to the averaged areal mass of the unit cell $\left(m_{0} /\left(L_{p}+W_{f}\right)^{2}\right)$. 


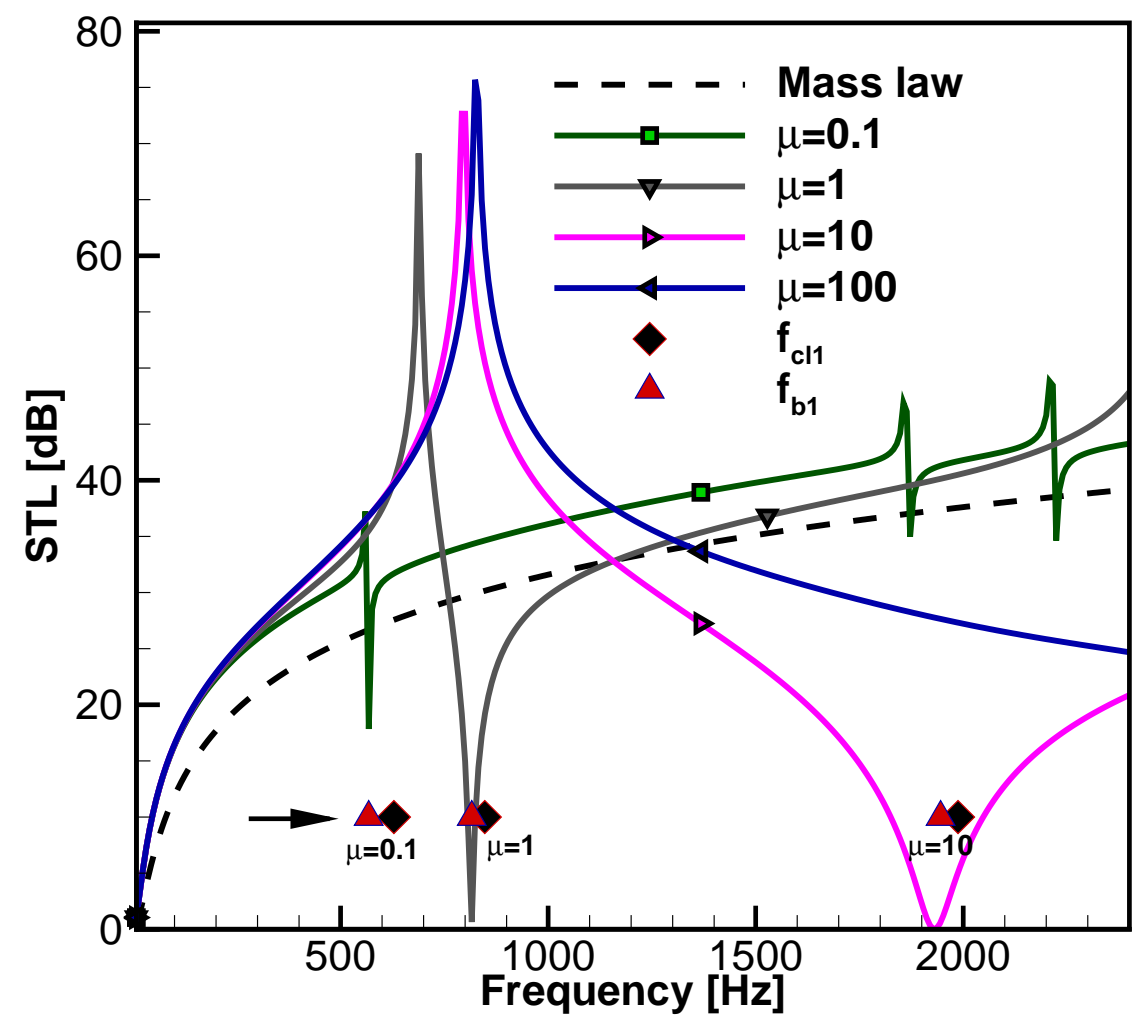

Figure 7: STL characteristics of cellular panels designed through materialbased mass apportioning, comparison to the first flexural eigenfrequency $f_{b 1}$, the first flexural mode frequency of a fully clamped plate- $f_{c l 1}$ (Eq. 4) for the cases of $\mu=0.1,1,10$, and the predicted STL for a mass law (Eq. 5) panel having an areal mass equal to the averaged areal mass of the unit cell $\left(m_{0} /\left(L_{p}+W_{f}\right)^{2}\right)$. 


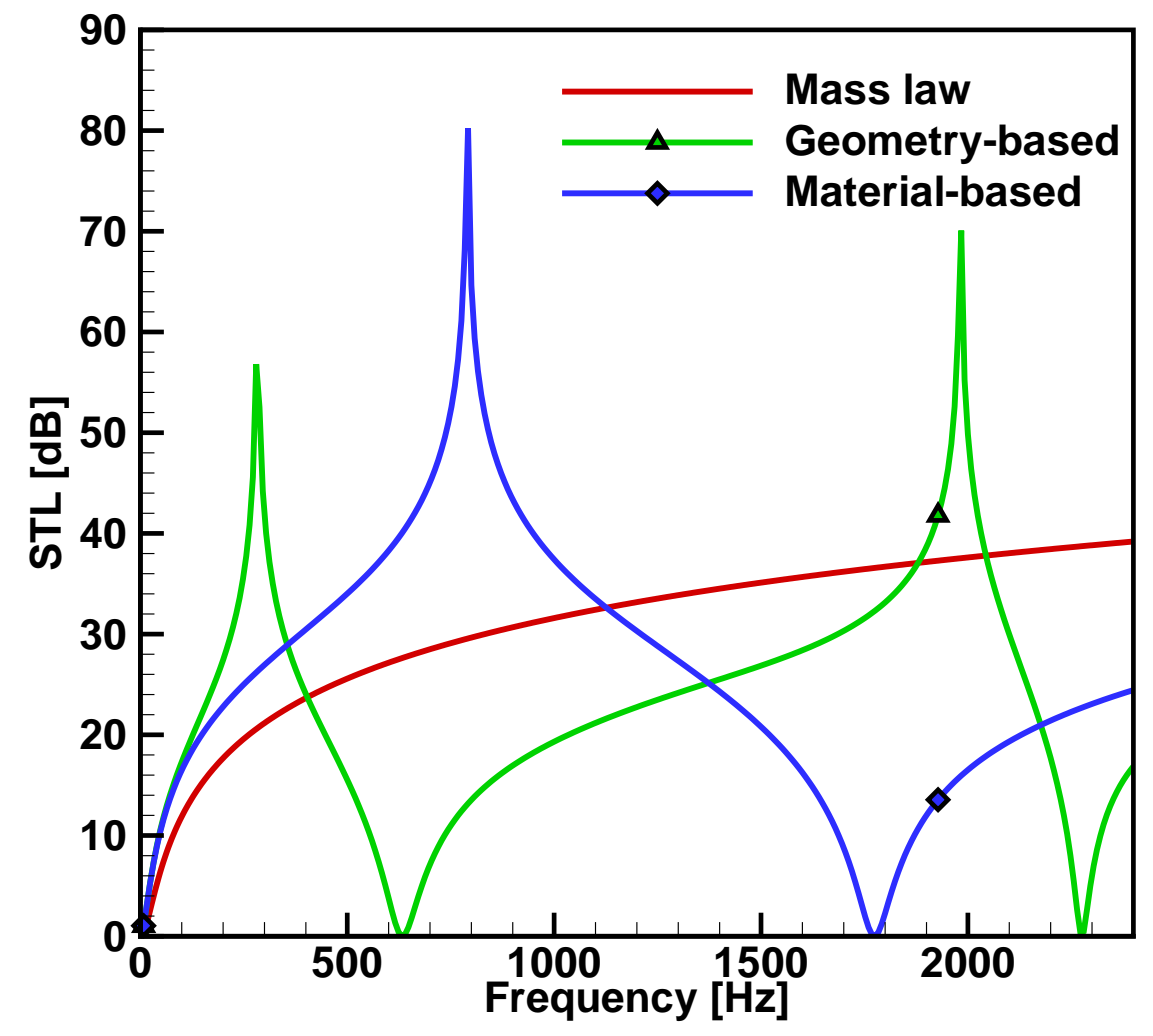

Figure 8: Comparison of predicted STL for material-based and geometrybased design approaches for $\mu=8.29$ and constant unit cell mass $m_{0}$ equal to that of the reference unit cell. Comparison to STL obtained for a mass law panel having an areal mass equal to the averaged areal mass of the unit cell $m_{0} /\left(L_{p}+W_{f}\right)^{2}$. 


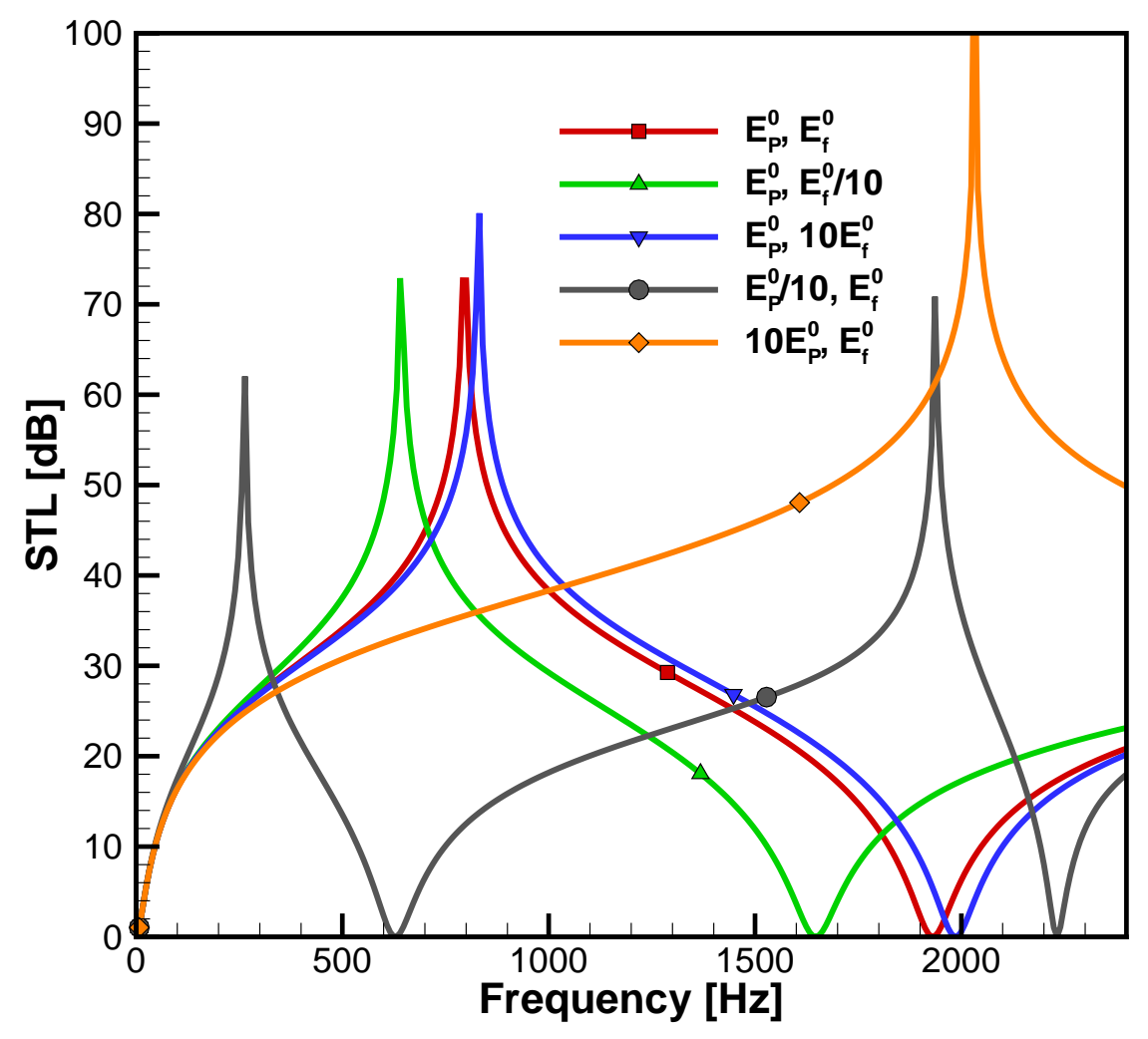

Figure 9: Effect of varying $E_{p}$ and $E_{f}$ on predicted STL while holding all the other material and geometric parameters of the unit cell constant $(\mu=10$ in material-based mass apportioning design). $E_{p}^{0}$ and $E_{f}^{0}$ are the unit cell material properties (see Table 2). 


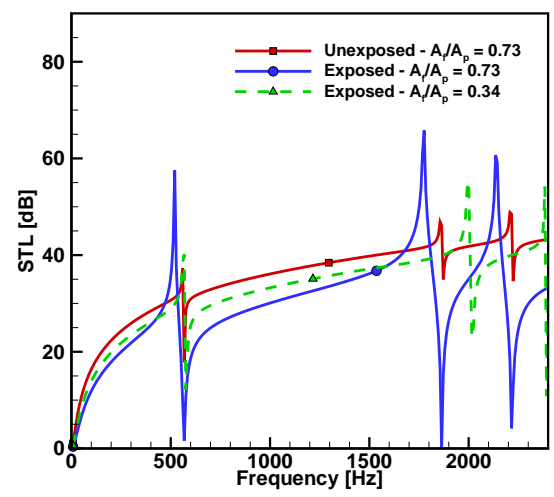

(a)

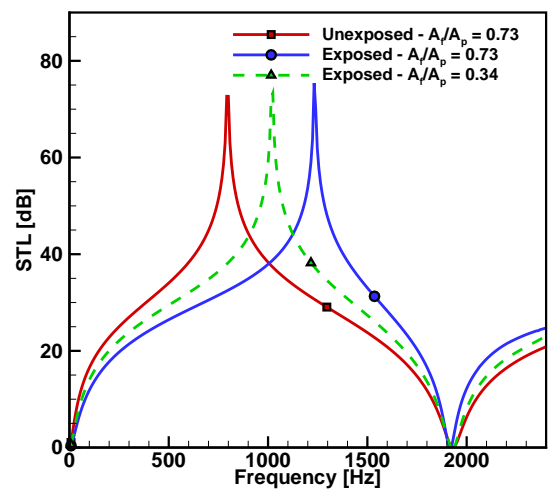

(c)

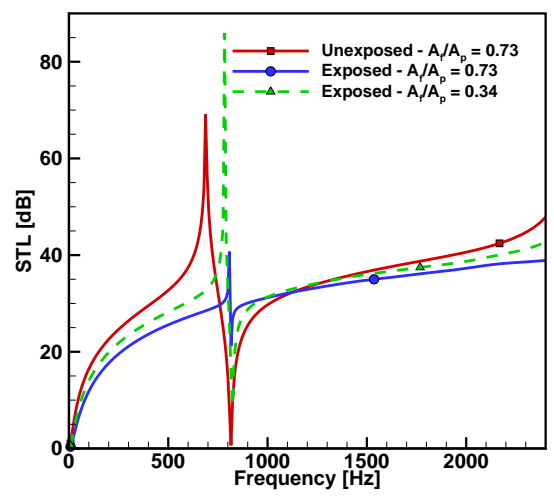

(b)

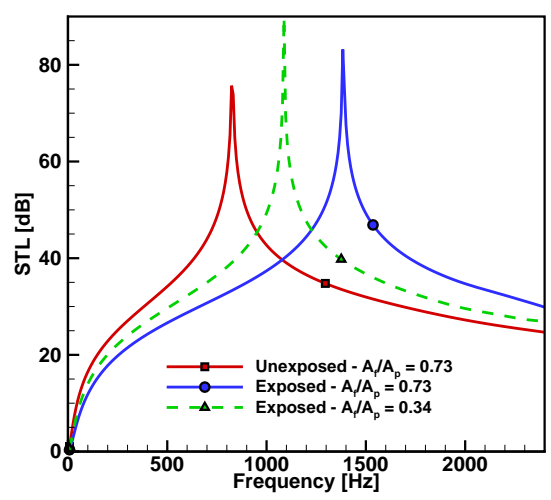

(d)

Figure 10: Effect of exposing the frame in the unit cell to acoustic loading on predicted STL for cases (a) $\mu=0.1$, (b) $\mu=1$, (c) $\mu=10$, and (d) $\mu=100$ in material-based mass apportioning design. 


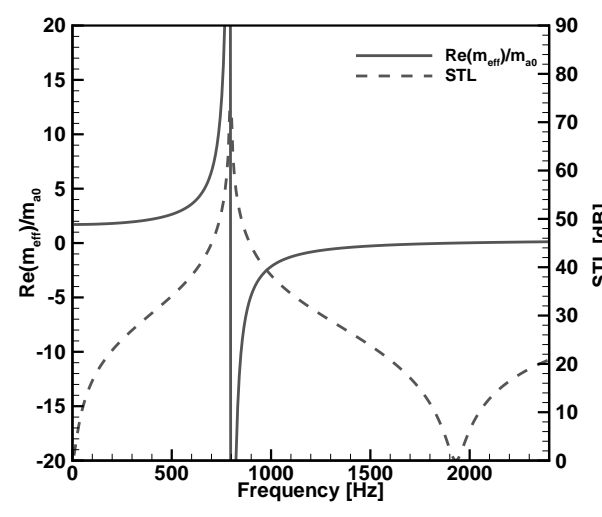

(a)

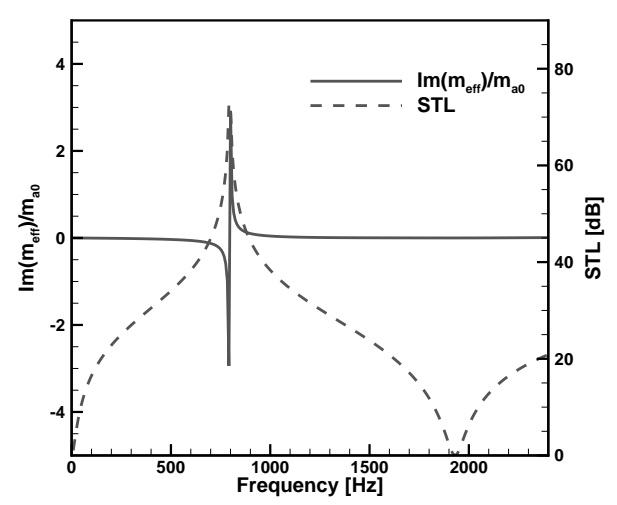

(b)

Figure 11: Real (a) and imaginary (b) parts of the effective mass (Eq. 6) as a function of frequency, and in comparison to the corresponding STL for the case of $\mu=10$ through material-based mass apportioning. Both the components are normalized using the averaged areal mass of the unit cell $m_{a 0}=m_{0} /\left(L_{p}+W_{f}\right)^{2}$ where $m_{0}$ is the total mass of the unit cell, and the other geometric parameters were defined in Eq. 1. 


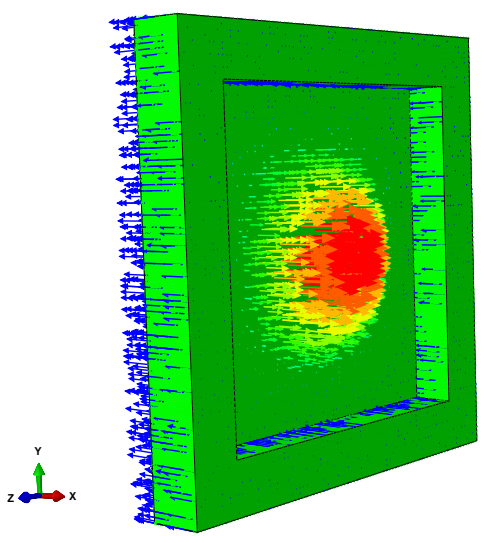

(a)

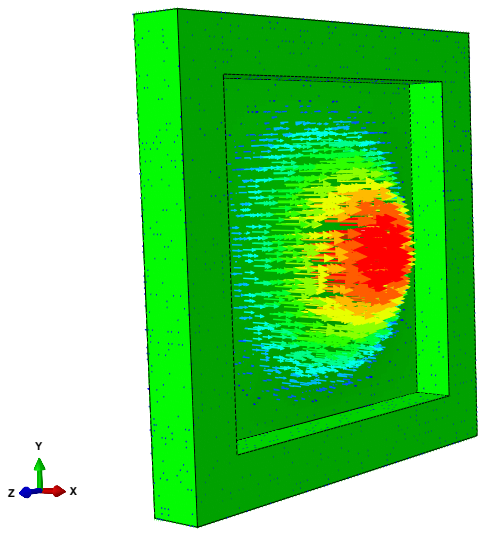

(b)

Figure 12: A qualitative comparison of predicted displacement in the $x$ direction (Re of $\operatorname{Re}+\jmath \mathrm{Im})$ at the (a) peak and (b) dip frequencies respectively of a unit cell in material-based approach with $\mu=10$. Red and blue indicate positive and negative displacements along the duct axis, respectively. 


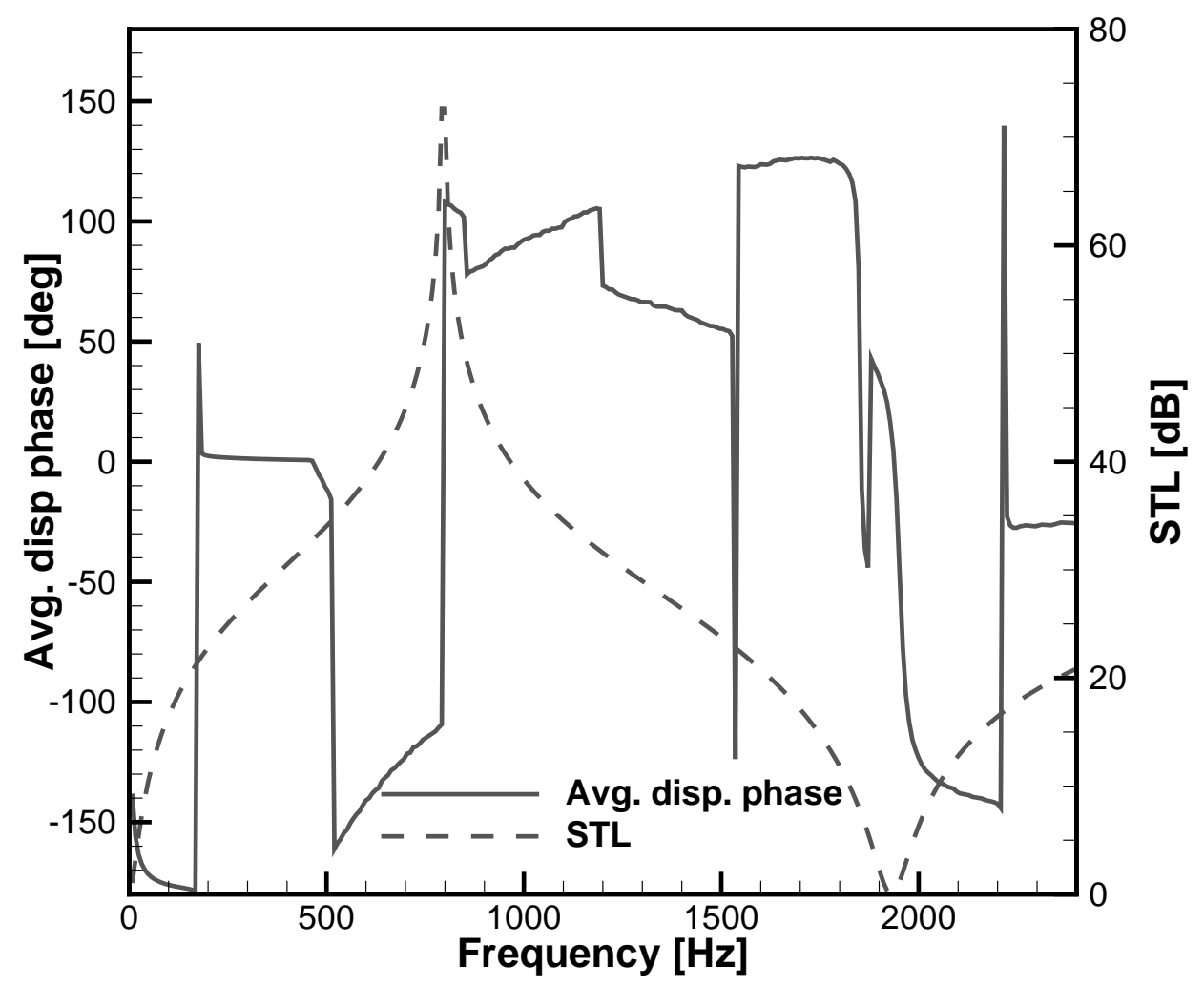

Figure 13: Averaged displacement phase for the case of $\mu=10$ in materialbased mass apportioning averaged over the plate region in the unit cell.

\section{References}

[1] R. Vaicaitis, M. Slazak, Noise transmission through stiffened panels, Journal of Sound and Vibration 70 (3) (1980) 413-426.

[2] J. P. Dowling, Sonic band-structure in fluids with periodic density variations, Journal of the Acoustical Society of America 91 (5) (1992) 2539-2543.

[3] Z. Y. Liu, X. X. Zhang, Y. W. Mao, Y. Y. Zhu, Z. Y. Yang, C. T. Chan, P. Sheng, Locally resonant sonic materials, Science 289 (5485) (2000) 1734-1736. 
[4] R. Martinezsala, J. Sancho, J. V. Sanchez, V. Gomez, J. Llinares, F. Meseguer, Sound-attenuation by sculpture, Nature 378 (6554) (1995) 241-241.

[5] P. Sheng, X. X. Zhang, Z. Liu, C. T. Chan, Locally resonant sonic materials, Physica B-Condensed Matter 338 (1-4) (2003) 201-205.

[6] E. C. Wester, X. Brémaud, B. Smith, Meta-material sound insulation, Building Acoustics 16 (2009) 21-30.

[7] K. M. Ho, Z. Yang, X. X. Zhang, P. Sheng, Measurements of sound transmission through panels of locally resonant materials between impedance tubes, Applied Acoustics 66 (7) (2005) 751-765.

[8] ASTM, Standard test method for measurement of normal incidence sound transmission of acoustical materials based on the transfer matrix method, in: E2611-09, Philadelphia, Pa., 2009.

[9] Z. Yang, J. Mei, M. Yang, N. H. Chan, P. Sheng, Membrane-type acoustic metamaterial with negative dynamic mass, Physical Review Letters 101 (20) (2008) 204301.

[10] J. Song, J. S. Bolton, Modeling of membrane sound absorbers, Proceedings of InterNoise 2002 (2002) 740-745.

[11] J. Song, J. S. Bolton, Acoustical modeling of tensioned, permeable membranes, Proceedings of Noise-Con 2003 (2003) 965-970.

[12] C. J. Naify, C. M. Chang, G. McKnight, S. Nutt, Transmission loss and dynamic response of membrane-type locally resonant acoustic metamaterials, Journal of Applied Physics 108 (11) (2010) 114905.

[13] Z. Yang, H. M. Dai, N. H. Chan, G. C. Ma, P. Sheng, Acoustic metamaterial panels for sound attenuation in the 50-1000 Hz regime, Applied Physics Letters 96 (4) (2010) 041906.

[14] H. H. Huang, C. T. Sun, Wave attenuation mechanism in an acoustic metamaterial with negative effective mass density, New Journal of Physics 11 (2009) 013003.

[15] X. M. Zhou, G. K. Hu, Analytic model of elastic metamaterials with local resonances, Physical Review B 79 (19) (2009) 195109.

[16] J. Mei, Z. Liu, W. Wen, P. Sheng, Effective dynamic mass density of composites, 
Physical Review B 76 (13) (2007) 134205.

[17] P. M. Morse, K. U. Ingard, Theoretical Acoustics, McGraw-Hill Book Company, Inc., New York, NY, 1968, Ch. 10, pp. 642-661.

[18] J. H. Lee, J. Kim, Analysis of sound transmission through periodically stiffened panels by space-harmonic expansion method, Journal of Sound and Vibration 251 (2) (2002) 349-366.

[19] L. L. Beranek (Ed.), Noise Reduction, McGraw-Hill Book Company, Inc., New York, NY, 1960, Ch. 13, pp. 280-302.

[20] B. H. Song, J. S. Bolton, Y. J. Kang, Effect of circumferential edge constraint on the acoustical properties of glass fiber materials, Journal of the Acoustical Society of America 110 (6) (2001) 2902-2916.

[21] B. H. Song, J. S. Bolton, Enhancement of the barrier performance of porous linings by using internal constraints, Noise Control Engineering Journal 51 (1) (2003) 1635 .

[22] SIMULIA, Inc., Providence, RI, USA, ABAQUS 6.10 documentation (May 2010).

[23] B. H. Song, J. S. Bolton, A transfer-matrix approach for estimating the characteristic impedance and wave numbers of limp and rigid porous materials, Journal of the Acoustical Society of America 107 (3) (2000) 1131-1152.

[24] J. S. Bolton, T. Yoo, O. Olivieri, Measurement of normal incidence transmission loss and other acoustical properties of materials placed in a standing wave tube, Tech. Rep. 1, Brüel and Kjær (2007).

[25] G. B. Warburton, The vibration of rectangular plates, Proc J Mech Engrs 168 (12) (1954) 371-384.

[26] V. Fokin, M. Ambati, C. Sun, X. Zhang, Method for retrieving effective properties of locally resonant acoustic metamaterials, Physical Review B 76 (14) (2007) 144302. 


\section{Appendix A. Experimental Validation}

Experiments were conducted using a modified Brüel and Kjær, fourmicrophone standing wave tube setup [8, 24] (see Fig. A.1)(a), and were used to validate the simulation set-up. The latter device is used in standardized test procedures to characterize barrier performance. The device consisted of two ducts with square cross-sections having an edge-length of $63.5 \mathrm{~mm}$, a sound source, and a data acquisition set-up. The end of the downstream duct was treated anechoically. The test sample was placed between the two ducts and was firmly held in-place by the ducts (see Fig. A.1)(b), which could be locked into position. To eliminate leakage of sound at the edges of the sample, two rubber gaskets $(2.0 \mathrm{~mm}$ thick) were applied to the end faces of the ducts that were in contact with the test sample. White noise was delivered to the loudspeaker and the pressure data was recorded at four microphone locations, two in the upstream and another two in the downstream duct section. The transfer functions between the first microphone and the three other microphones were estimated and were then used to evaluate the STL of the sample in exactly the same way as in the finite element simulations [23].

The test sample used in the experiment was made using extruded acrylic (PMMA), the material properties of which, namely, the elastic modulus and material density can vary in the ranges of $2.24-3.38 \mathrm{GPa}$, and $1162-1190$ $\mathrm{kg} / \mathrm{m}^{3}$, respectively, as specified by the vendor (McMaster-Carr). The shape of the test specimen was the same as the unit cell (see Fig. 1). Its dimensions are: (1) $t_{p}=1.52 \mathrm{~mm},(2) t_{f}=9.06 \mathrm{~mm},(3) L_{p}=63.5 \mathrm{~mm}$, and $W_{f}=$ 
$10 \mathrm{~mm}$ where the definitions of the geometric parameters $t_{p}, t_{f}, L_{p}$ and $W_{f}$ are the same as specified in Eq. 1 and as labeled in Fig. 1(b). The test specimen was made by machining the frame and the plate from sheets of thickness 7.52 and $1.524 \mathrm{~mm}$ respectively, and fusing them using a chemical cement (Weld-On 3). This route was chosen to make the test sample since machining a single piece test specimen from an acrylic sheet is not feasible owing to the low thickness $\left(t_{p}\right)$ required for the plate.

The simulation setup described in Section 3 was used to reproduce the experimentally predicted STL, with some modifications. Unlike the simulations of a unit cell in the cellular metamaterial, the presence of soft-rubber gaskets holding the sample in-place in the experimental set-up was simulated in the numerical model by attaching a gasket of $1 \mathrm{~mm}$ thickness to either side of the frame, normal to the duct axis. To mimic the boundary conditions seen by the test sample in the experiments (see Fig. A.1(b)), all the nodes on the gasket/duct interfaces were constrained to have zero displacement in all directions. This model with the above boundary conditions was used only to compare with the experimental results as noted in the main body of this article. Boundary conditions enforcing spatial periodicity were imposed in the other simulations reported in the main text. The numerical simulation here was performed using a material density of $1162 \mathrm{~kg} / \mathrm{m}^{3}$, an elastic modulus of $3.72 \mathrm{GPa}(10 \%$ higher than the modulus specified by the vendor), and a poisson ratio of 0.43 . The modulus of the gasket was taken to be $5 \mathrm{MPa}$ which is a typical value for Silicone Rubber elastomer and the poisson ratio was set to 0.45 . Rayleigh damping was incorporated both into 
the gasket and the specimen with the mass-proportional damping, $\alpha$, equal to 300 and the stiffness proportional damping, $\beta$, equal to $0.3 \times 10^{-5}$.

Experimental data from 12 runs was used to obtain the mean and the standard deviation of the STL. The experimental results were compared with the predicted STL in Fig. A.2. Note that the results below $200 \mathrm{~Hz}$ are entirely dominated by noise effects which typically are significant at low frequencies in measurements of this type, particularly when STL is large [24].

From Fig. A.2, it can be seen that the simulation setup accurately reproduces the experimental features: It reflects the non-zero STL at region close to $0 \mathrm{~Hz}$, captures the dropping trend of STL from a high value to a very low value at the dip, qualitatively and quantitatively, and then increases subsequently in-line with the experimental results. At the dip, it can be seen that the simulation model predicts the STL accurately. Figure A.2 also shows the predicted STL for a simulation without damping, in which case the STL goes to zero at the dip. Except for the non-zero STL at the dip, there is no change in the predicted characteristics due to the presence of damping. The results presented in the main body of the paper were obtained without including damping to simplify the analysis process. 


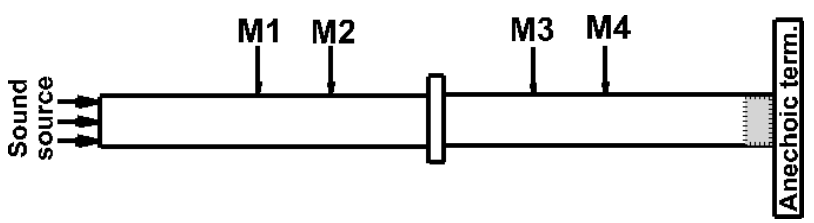

(a)

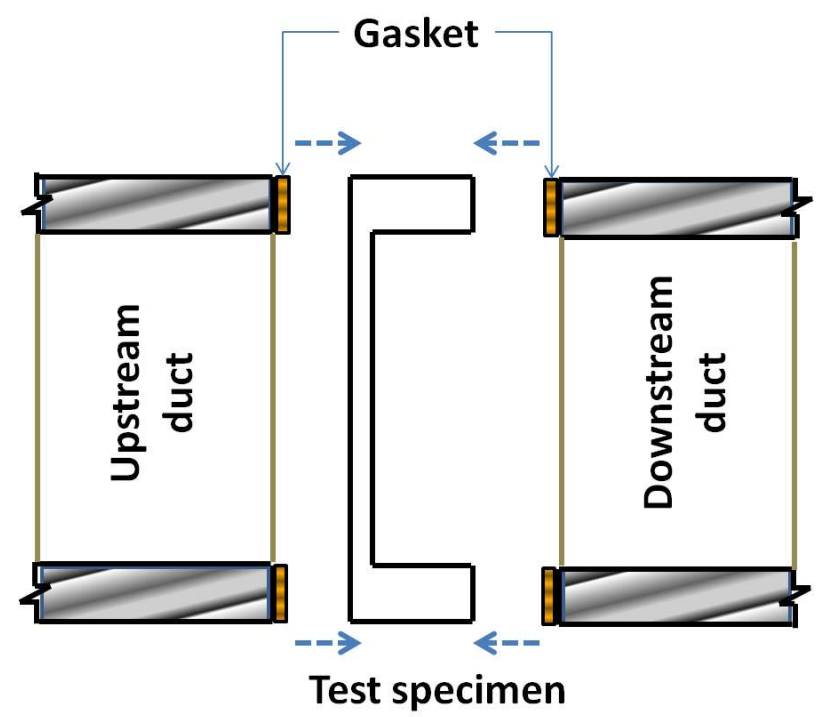

(b)

Figure A.1: (a) A schematic showing the standard Standing Wave Tube Setup [8] used for characterizing barrier materials, and (b) a schematic drawing illustrating the placement of test specimen in the standing wave tube set-up and the need for imposing the specified boundary conditions for experimental validation. 


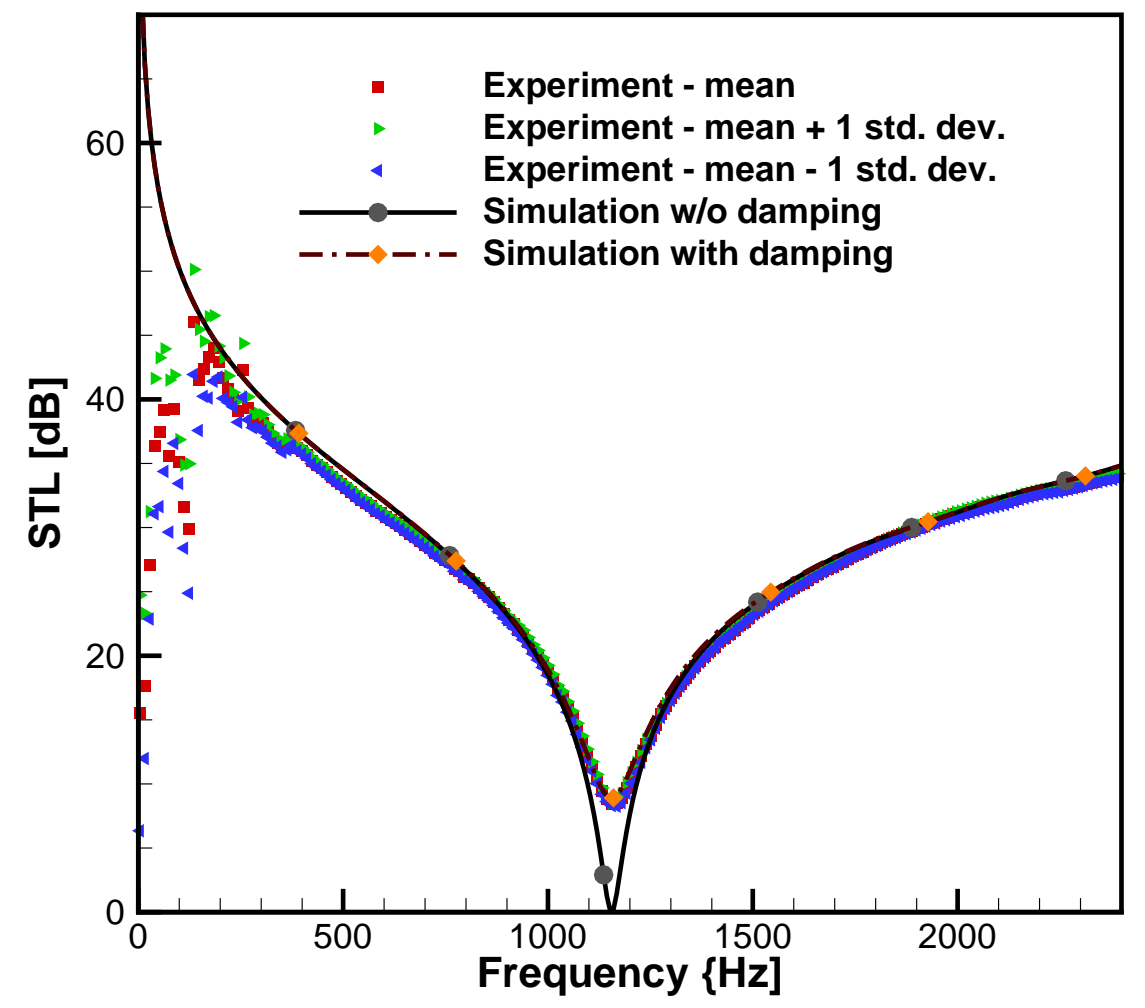

Figure A.2: Comparison of predicted and measured STLs for an extruded acrylic test sample. 


\section{Appendix B. Mesh convergence studies}

Mesh convergence studies were performed on a flat square panel with an edge length of $63.5 \mathrm{~mm}$, and having a uniform thickness of $2 \mathrm{~mm}$. Its lateral faces were completely constrained from moving in any direction and a 20-node quadratic hexahedral element was chosen for the element type. An edge-constrained plate behavior was expected for this model. The study was performed by varying the number of layers of elements within the thickness while keeping the in-plane element size constant to assess the dependence of the predicted result on the thickness of the element. Similarly, the dependence of the predicted STL on the in-plane element size was assessed by varying the number of elements while requiring their in-plane aspect ratio to be one. Figure B.1 shows the predicted STL characteristics for the panel with different element sizes. No significant change in the predicted STL was observed in any case. This indicates that the predicted STL was independent of the element resolution when the number of layers of elements were more than three, and the number of elements on each edge was greater than 32. 


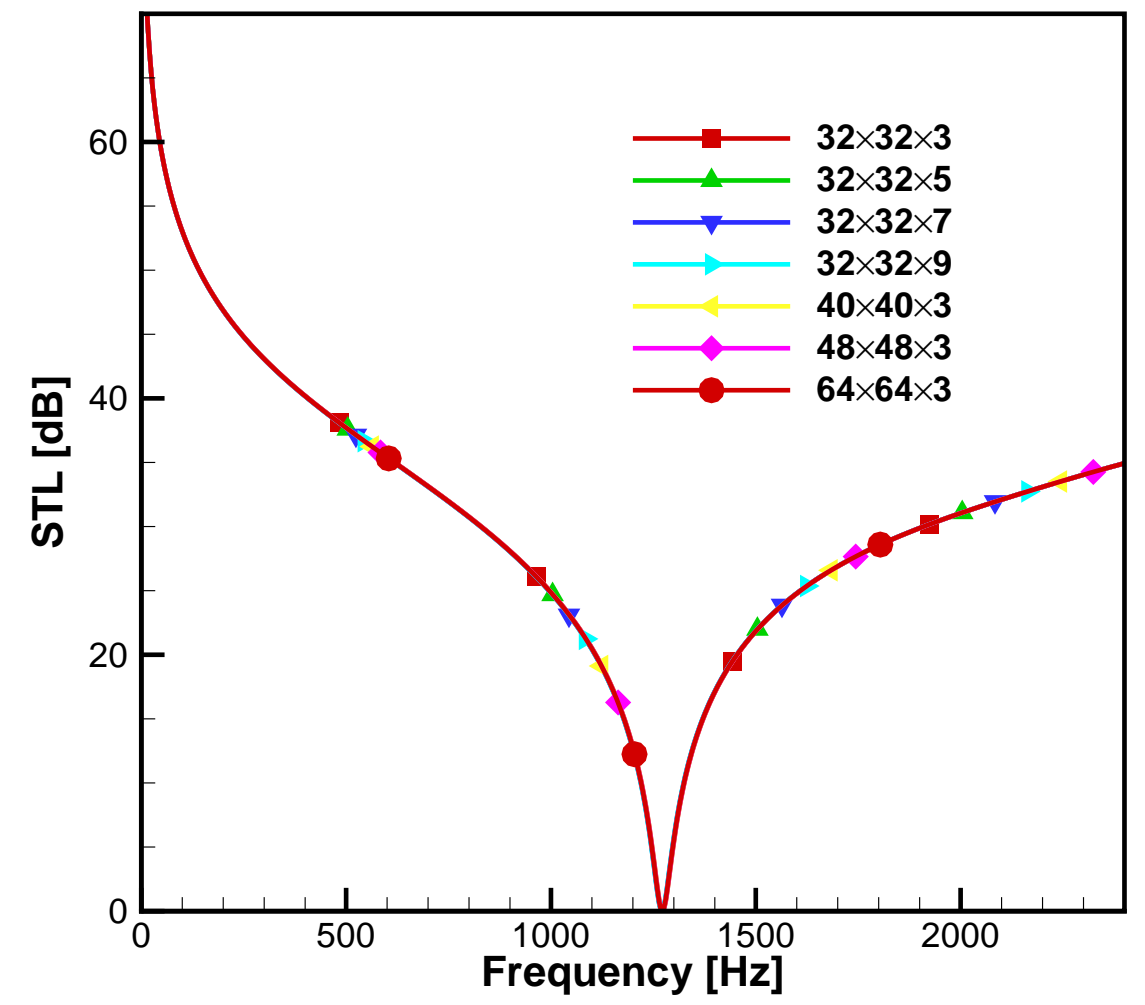

Figure B.1: Mesh convergence study for different element sizes in the solid domain of the simulation setup. 